\title{
TUNGSTEN ISOTOPIC COMPOSITIONS IN STARDUST SiC GRAINS FROM THE MURCHISON METEORITE: CONSTRAINTS ON THE $s$-PROCESS IN THE Hf-Ta-W-Re-Os REGION
}

\author{
Janaína N. Ávila ${ }^{1,2,7}$, Maria Lugaro ${ }^{3}$, Trevor R. Ireland ${ }^{1,2}$, Frank Gyngard ${ }^{4}$, Ernst Zinner ${ }^{4}$, Sergio Cristallo $^{5,8}$, \\ Peter Holden $^{1}$, Joelene Buntain $^{3}$, Sachiko Amari $^{4}$, and Amanda KaraKas 6 \\ ${ }^{1}$ Research School of Earth Sciences, The Australian National University, Canberra, ACT 0200, Australia; janaina.avila@ anu.edu.au \\ ${ }^{2}$ Planetary Science Institute, The Australian National University, Canberra, ACT 0200, Australia \\ ${ }^{3}$ Centre for Stellar and Planetary Astrophysics, Monash University, Clayton, VIC 3800, Australia \\ ${ }^{4}$ Laboratory for Space Sciences and the Department of Physics, Washington University, One Brookings Drive, St. Louis, MO 63130, USA \\ ${ }^{5}$ Departamento de Física Teórica y del Cosmos, Universidad de Granada, Granada 18071, Spain \\ ${ }^{6}$ Mount Stromlo Observatory, Australian National University, Weston Creek, ACT 2611, Australia \\ Received 2011 June 24; accepted 2011 October 8; published 2011 December 13
}

\begin{abstract}
We report the first tungsten isotopic measurements in stardust silicon carbide ( $\mathrm{SiC}$ ) grains recovered from the Murchison carbonaceous chondrite. The isotopes ${ }^{182,183,184,186} \mathrm{~W}$ and ${ }^{179,180} \mathrm{Hf}$ were measured on both an aggregate (KJB fraction) and single stardust $\mathrm{SiC}$ grains (LS+LU fraction) believed to have condensed in the outflows of low-mass carbon-rich asymptotic giant branch (AGB) stars with close-to-solar metallicity. The SiC aggregate shows small deviations from terrestrial (= solar) composition in the ${ }^{182} \mathrm{~W} /{ }^{184} \mathrm{~W}$ and ${ }^{183} \mathrm{~W} /{ }^{184} \mathrm{~W}$ ratios, with deficits in ${ }^{182} \mathrm{~W}$ and ${ }^{183} \mathrm{~W}$ with respect to ${ }^{184} \mathrm{~W}$. The ${ }^{186} \mathrm{~W} /{ }^{184} \mathrm{~W}$ ratio, however, shows no apparent deviation from the solar value. Tungsten isotopic measurements in single mainstream stardust $\mathrm{SiC}$ grains revealed lower than solar ${ }^{182} \mathrm{~W} /{ }^{184} \mathrm{~W},{ }^{183} \mathrm{~W} /{ }^{184} \mathrm{~W}$, and ${ }^{186} \mathrm{~W} /{ }^{184} \mathrm{~W}$ ratios. We have compared the $\mathrm{SiC}$ data with theoretical predictions of the evolution of $\mathrm{W}$ isotopic ratios in the envelopes of AGB stars. These ratios are affected by the slow neutron-capture process and match the $\mathrm{SiC}$ data regarding their ${ }^{182} \mathrm{~W} /{ }^{184} \mathrm{~W},{ }^{183} \mathrm{~W} /{ }^{184} \mathrm{~W}$, and ${ }^{179} \mathrm{Hf} /{ }^{180} \mathrm{Hf}$ isotopic compositions, although a small adjustment in the $s$-process production of ${ }^{183} \mathrm{~W}$ is needed in order to have a better agreement between the $\mathrm{SiC}$ data and model predictions. The models cannot explain the ${ }^{186} \mathrm{~W} /{ }^{184} \mathrm{~W}$ ratios observed in the $\mathrm{SiC}$ grains, even when the current ${ }^{185} \mathrm{~W}$ neutron-capture cross section is increased by a factor of two. Further study is required to better assess how model uncertainties (e.g., the formation of the ${ }^{13} \mathrm{C}$ neutron source, the mass-loss law, the modeling of the third dredge-up, and the efficiency of the ${ }^{22} \mathrm{Ne}$ neutron source) may affect current $s$-process predictions.
\end{abstract}

Key words: dust, extinction - nuclear reactions, nucleosynthesis, abundances - stars: AGB and post-AGB - stars: carbon

\section{INTRODUCTION}

Stardust grains condensed directly from the gas phase present in ancient stellar outflows or stellar ejecta and thus became part of the interstellar medium from which our solar system formed about 4.57 Gyr ago. Their stellar origins are indicated by unusual isotopic compositions (essentially for every element) relative to those found in solar system materials (i.e., terrestrial, lunar, and meteoritic samples). The deviations from the recommended solar system isotopic abundances, which are taken from compilations of isotopic measurements of terrestrial materials except for H and the noble gases (e.g., Anders \& Grevesse 1989; Lodders 2003,2010 ), are too large to be explained by mass-fractionation processes or by decay of longer-lived radioactive isotopes. Instead, the observed isotopic compositions can only be explained by nucleosynthetic processes (see reviews by Zinner 1998, 2004; Clayton \& Nittler 2004; Lodders \& Amari 2005).

Isotopic compositions of several heavy elements have been measured in stardust grains believed to have condensed in the outflows of low-mass carbon-rich asymptotic giant branch (AGB) stars (e.g., Zr, Nicolussi et al. 1997; Mo, Nicolussi et al. 1998a; Sr, Nicolussi et al. 1998b; Ba, Savina et al. 2003; $\mathrm{Ru}$, Savina et al. 2004). The grain data have provided detailed

\footnotetext{
7 Current address: Astronomy Department/IAG, University of São Paulo, São Paulo, SP 05508-090, Brazil.

8 Current address: Osservatorio Astronomico di Collurania, INAF, Teramo 64100, Italy.
}

information on the nucleosynthesis of heavy elements produced by the slow neutron capture process (the $s$-process). However, the available data are still scarce or no data exist for some heavy elements. One of the main gaps involves nuclides in the Hf-Ta-W-Re-Os region.

The $s$-process path in the Hf-Ta-W-Re-Os region (Figure 1) has recently received considerable attention. New experimental data (neutron-capture reaction rates) for $\mathrm{Hf}, \mathrm{W}$, and Os nuclides have been reported (Sonnabend et al. 2003; Mohr et al. 2004; Mosconi et al. 2006, 2010a, 2010b; Wisshak et al. 2006; Segawa et al. 2007; Vockenhuber et al. 2007; Marganiec et al. 2009; Fujii et al. 2010), and small anomalies of nucleosynthetic origin in $\mathrm{W}$ and Os isotopes have been observed in primitive meteorites (Brandon et al. 2005; Yokoyama et al. 2007, 2011; Qin et al. 2008; Reisberg et al. 2009). Recent $s$-process analyses of the Hf-Ta-W-Re-Os region have identified two major problems. First, it appears that model predictions underestimate the $s$-process contribution to the ${ }^{182} \mathrm{~W}$ solar abundance and, consequently, the ${ }^{182} \mathrm{~W} r$-residual (obtained by subtracting the calculated $s$-process from the observed solar system abundance) shows a significant positive deviation from the otherwise very smooth rapid neutron-capture process (the $r$-process) solar abundance pattern (see Figure 12 of Wisshak et al. 2006; Figure 6 of Vockenhuber et al. 2007). Second, an analysis of the $s$-process flow at the ${ }^{185} \mathrm{~W}$ branching point shows that the predicted ${ }^{186} \mathrm{Os} s$-process abundance is also somewhat problematic, suggesting a significant overproduction 


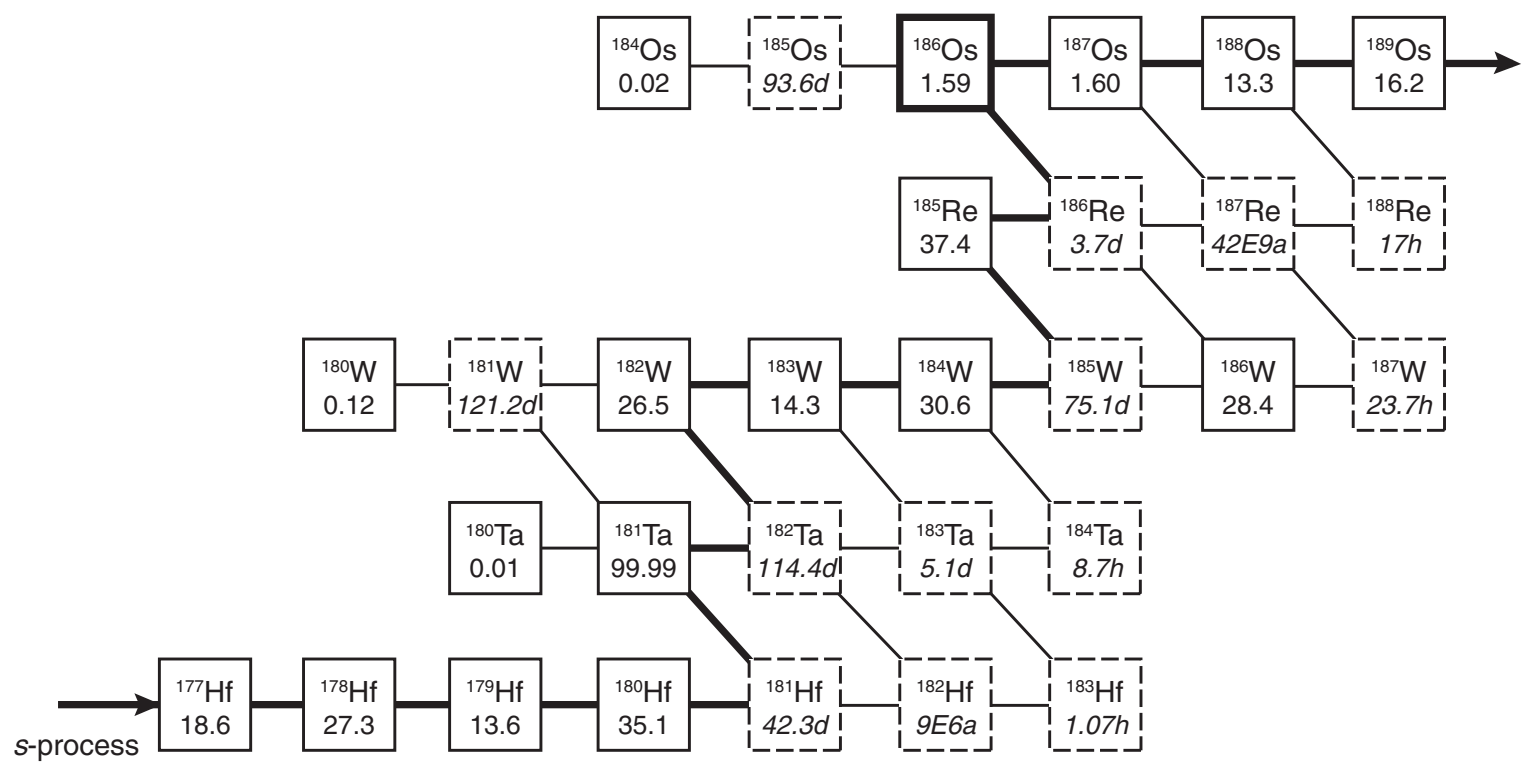

Figure 1. Part of the nuclide chart showing the $s$-process nucleosynthesis path in the region of Hf-Ta-W-Re-Os (modified from Hayakawa et al. 2005; and Dillmann et al. 2006). Percent abundances (non-italic) are shown for each stable isotope (solid boxes) and laboratory half-lives (italic) for each unstable isotope (dashed line boxes). Half-lives at stellar temperatures may be different, as discussed in the text. The main $s$-process path is shown as a bold line and branches and secondary paths are shown as finer lines, $s$-only isotope ${ }^{186} \mathrm{Os}$ is indicated by a bold box.

( $\sim 20 \%$ ) with respect to its solar abundance (Sonnabend et al. 2003). This is not allowed, theoretically, because ${ }^{186}$ Os is an $s$-only isotope, being shielded by the stable ${ }^{186} \mathrm{~W}$ from the chain of radioactive decays that follow the $r$-process. In line with this, isotopic anomalies of nucleosynthetic origin found in Os isotopes measured in primitive meteorites suggest a lower ${ }^{186} \mathrm{Os} /{ }^{188} \mathrm{Os} s$-process ratio than current models predict (Brandon et al. 2005; Yokoyama et al. 2007). The problematic ${ }^{182} \mathrm{~W}$ and ${ }^{186} \mathrm{Os} s$-process abundances and, consequently, the inferred ${ }^{182} \mathrm{~W} r$-residual, may reflect remaining uncertainties related to the experimental cross section data and the current $s$-process models.

Tungsten has five stable isotopes: the rare ${ }^{180} \mathrm{~W}(0.12 \%$ of solar W), which is mainly produced by proton capture or photodisintegration processes ( $p$-process), and ${ }^{182} \mathrm{~W}(26.5 \%)$, ${ }^{183} \mathrm{~W}(14.3 \%),{ }^{184} \mathrm{~W}(30.6 \%)$, and ${ }^{186} \mathrm{~W}(28.4 \%)$, which are of mixed $s$ - and $r$-process origin. As illustrated in Figure 1, the branchings at ${ }^{181,182} \mathrm{Hf}$ and ${ }^{182,183} \mathrm{Ta}$ may affect the W $s$-process isotopic pattern, and the branching at ${ }^{185} \mathrm{~W}$ determines the ${ }^{186} \mathrm{~W}$ abundance and may affect the Re and Os isotopic patterns.

Here, we report the results of the first $\mathrm{W}$ isotopic analyses performed on five large stardust silicon carbide ( $\mathrm{SiC}$ ) grains (LS+LU fraction) extracted from the Murchison carbonaceous chondrite (Amari et al. 1994). In addition to the individual grains, we also analyzed an SiC-enriched bulk sample (KJB fraction; Amari et al. 1994). Carbon-, $\mathrm{N}$-, and $\mathrm{Si}$-isotopic ratios for the KJB fraction and individual $\mathrm{SiC}$ grains from the $\mathrm{LS}+\mathrm{LU}$ fraction have been previously reported by Amari et al. (2000) and Virag et al. (1992), and are reproduced in Figure 2 and Table 1. The mount containing grains from the LS+LU fraction used in this study is the same previously investigated by Virag et al. (1992) and Ireland et al. (1991). Virag et al. (1992) have shown that SiC grains from the LS+LU fraction contain several unique features: some are very large (over $20 \mu \mathrm{m}$ ); many of these large grains appear to have flat and smooth surfaces, unlike the euhedral surfaces observed in smaller grains; and isotopic compositions show clustering for $\mathrm{C}$ and $\mathrm{Si}$ and even for $\mathrm{Ti}$ (Ireland et al. 1991). Recent studies have shown that stardust
$\mathrm{SiC}$ grains from the $\mathrm{LS}+\mathrm{LU}$ fraction have interstellar exposure ages ranging from $\sim 3 \mathrm{Myr}$ to $\sim 1 \mathrm{Gyr}$ (Gyngard et al. 2009a, 2009b; Heck et al. 2009), which implies that the parent stars of the grains must have ended their lives within this time range before the formation of the solar system. Based on their C-, N-, and $\mathrm{Si}$-isotopic compositions, the five single $\mathrm{SiC}$ grains analyzed for W are classified as "mainstream grains," and are interpreted to have condensed in the outflows of low-mass $\left(\sim 1.5-3 M_{\odot}\right)$, carbon-rich AGB stars with close-to-solar metallicity (Hoppe et al. 1994; Zinner et al. 2006). The KJB fraction also shows $\mathrm{C}-, \mathrm{N}$-, and $\mathrm{Si}$-isotopic signatures consistent with an AGB origin for most of the grains (Amari et al. 2000).

\section{TUNGSTEN ISOTOPIC MEASUREMENTS}

While ${ }^{182} \mathrm{~W}$ and ${ }^{183} \mathrm{~W}$ are free of interferences, the remaining $\mathrm{W}$ isotopes have atomic isobaric interferences: ${ }^{180} \mathrm{~W}$ from ${ }^{180} \mathrm{Ta}^{+}\left(0.012 \%\right.$ of solar Ta) and ${ }^{180} \mathrm{Hf}^{+}(35.1 \%$ of solar $\mathrm{Hf})$, ${ }^{184} \mathrm{~W}$ from ${ }^{184} \mathrm{Os}^{+}(0.02 \%$ of solar Os $)$, and ${ }^{186} \mathrm{~W}$ from ${ }^{186} \mathrm{Os}^{+}$ (1.59\% of solar Os). Monoxide interferences are also present (e.g., ${ }^{170} \mathrm{Er}^{16} \mathrm{O}^{+}$and ${ }^{170} \mathrm{Yb}^{16} \mathrm{O}^{+}$on ${ }^{186} \mathrm{~W}^{+}$), requiring a mass resolution of $m / \Delta m \sim 8000$ for separation. Detailed scans in the mass region of $\mathrm{W}^{+}$and $\mathrm{WO}^{+}$isotopic species, sputtered from NIST-610 silicate glass and an SiC ceramic doped with heavy elements, have shown that $\mathrm{WO}^{+}$species are produced at a higher intensity than $\mathrm{W}^{+}$species during sputtering by a $10 \mathrm{keV} \mathrm{O}_{2}^{-}$primary ion beam $\left(\mathrm{WO}^{+} / \mathrm{W}^{+} \sim 3\right)$. Furthermore, as previously described by Kinny et al. (1991), under the same analytical conditions $\mathrm{YbO}^{+} / \mathrm{Yb}^{+} \sim 0.5$, and $\mathrm{REEO}_{2}^{+} / \mathrm{REEO}^{+}$is negligible $(<0.001)$. Analyzing $\mathrm{WO}^{+}$instead of $\mathrm{W}^{+}$therefore produces higher yields and minimizes interferences from $\mathrm{REE}^{+}, \mathrm{REEO}^{+}$, and $\mathrm{REEO}_{2}{ }^{+}$species. For these reasons we analyzed $\mathrm{W}$ isotopes as $\mathrm{WO}^{+}$.

Tungsten isotopic measurements in stardust $\mathrm{SiC}$ grains were carried out with a Sensitive High Resolution Ion MicroprobeReverse Geometry (SHRIMP-RG) at the Australian National University. We performed both "bulk analyses" on an aggregate of many grains from the KJB fraction and "single-grain analyses" on grains from the LS+LU fraction. Nine individual 

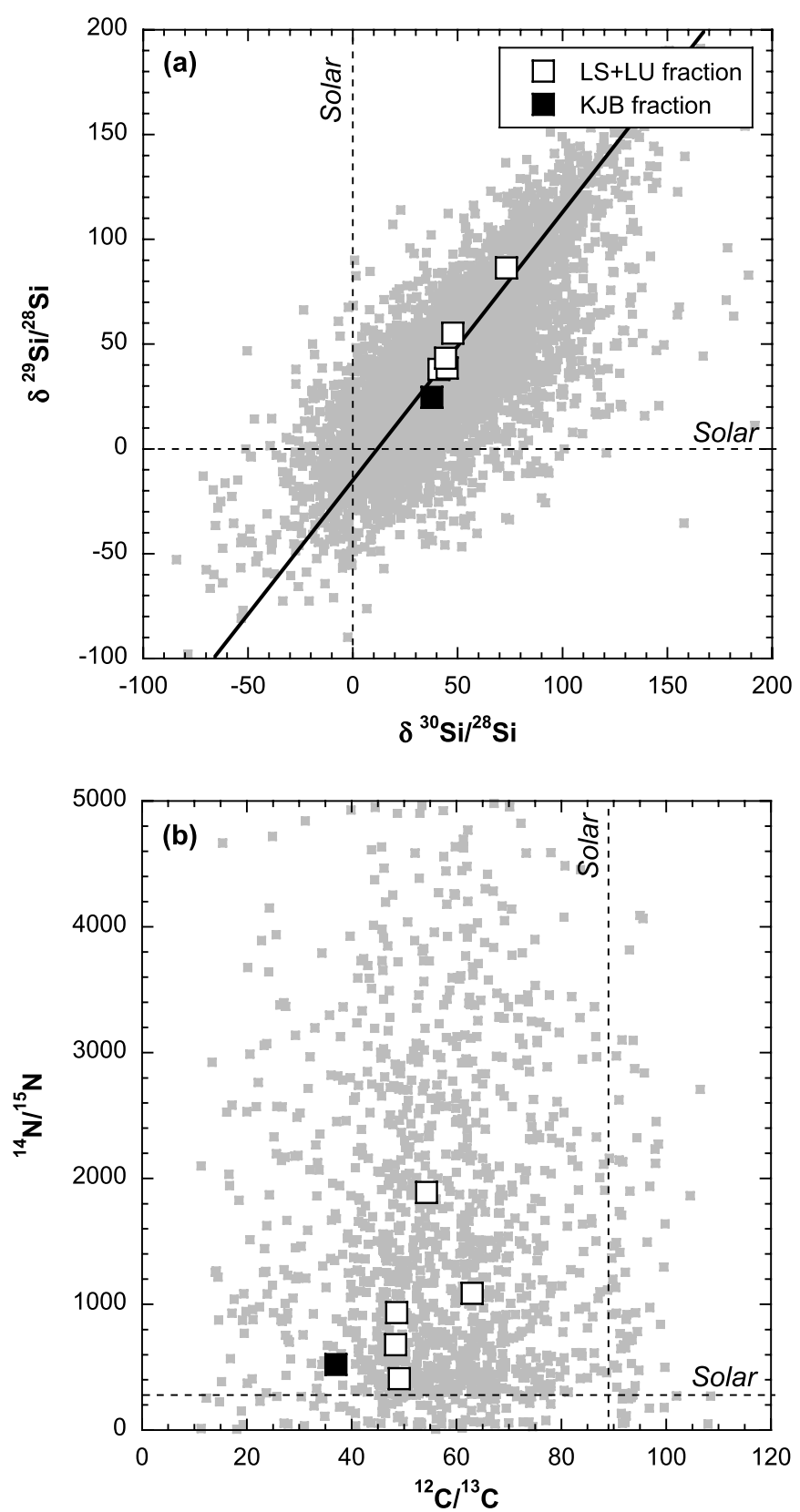

Figure 2. Silicon-, $\mathrm{C}-$, and $\mathrm{N}$-isotopic ratios of stardust $\mathrm{SiC}$ grains from the LS+LU fraction (white squares, from Virag et al. 1992) analyzed in the present study. Data for mainstream SiC grains from previous analyses (Hynes \& Gyngard 2009) are shown for comparison (gray squares). Data for the SiCenriched bulk sample (KJB fraction, black square) from Amari et al. (2000) are also plotted. Error bars from previous measurements are omitted for clarity. Black dashed lines indicate the solar ratios (Lodders 2010) as inferred from terrestrial composition. (a) $\mathrm{Si}$-isotopic ratios expressed as deviations ( $\delta$-values) from the reference terrestrial $(=$ solar $)$ isotopic composition in parts per thousand $(\%)$. Error bars are smaller than the symbols. Also shown is the so-called mainstream correlation line indicated by the solid line, with slope of 1.35 (Zinner et al. 2006). (b) ${ }^{14} \mathrm{~N} /{ }^{15} \mathrm{~N}$ ratios plotted against ${ }^{12} \mathrm{C} /{ }^{13} \mathrm{C}$ ratios. Error bars are smaller than the symbols.

spots on the KJB fraction were analyzed for $\mathrm{W}$ isotopes. Five out of nine investigated single grains from the LS+LU fraction had sufficiently high $\mathrm{W}$ concentrations for isotopic analysis. SHRIMP-RG measurements were performed with an $\mathrm{O}_{2}^{-}$primary beam of $\sim 5 \mathrm{nA}$ focused to sputter an area of $\sim 30 \mu \mathrm{m}$ in diameter. Before data acquisition, each spot/grain was initially rastered across an area slightly larger than the analytical pit by the beam for $\sim 60 \mathrm{~s}$ to minimize surface contamination. Secondary ions were extracted at $10 \mathrm{kV}$ and measured by single collector analysis on an $\mathrm{ETP}^{\mathrm{TM}}$ multiplier in peak-jumping mode.

We used two different setups. In the first setup (KJB spots $01-08$ ), we measured ${ }^{180} \mathrm{Hf}^{16} \mathrm{O}^{+},{ }^{182} \mathrm{~W}^{16} \mathrm{O}^{+},{ }^{183} \mathrm{~W}^{16} \mathrm{O}^{+}$, ${ }^{184} \mathrm{~W}^{16} \mathrm{O}^{+},{ }^{186} \mathrm{~W}^{16} \mathrm{O}^{+},{ }^{188} \mathrm{Os}^{16} \mathrm{O}^{+}$, and ${ }^{189} \mathrm{Os}^{16} \mathrm{O}^{+}$. We monitored $\mathrm{OsO}^{+}$in order to estimate a potential interference on the ${ }^{186} \mathrm{~W}^{16} \mathrm{O}^{+}$peak. However, no contribution was found in the mass regions of ${ }^{188} \mathrm{Os}^{16} \mathrm{O}^{+}$and ${ }^{189} \mathrm{Os}^{16} \mathrm{O}^{+}$. In the second setup (KJB spot 09, and all individual grains), we measured ${ }^{179} \mathrm{Hf}^{16} \mathrm{O}^{+},{ }^{180} \mathrm{Hf}^{16} \mathrm{O}^{+},{ }^{182} \mathrm{~W}^{16} \mathrm{O}^{+},{ }^{183} \mathrm{~W}^{16} \mathrm{O}^{+},{ }^{184} \mathrm{~W}^{16} \mathrm{O}^{+}$, ${ }^{186} \mathrm{~W}^{16} \mathrm{O}^{+}$, ${ }^{188} \mathrm{Os} \mathrm{s}^{16} \mathrm{O}^{+}$, and ${ }^{189} \mathrm{Os}^{16} \mathrm{O}^{+}$. Contributions from ${ }^{180} \mathrm{~W}^{16} \mathrm{O}^{+}$on the ${ }^{180} \mathrm{Hf}^{16} \mathrm{O}^{+}$peak, ${ }^{180} \mathrm{Hf}^{18} \mathrm{O}^{+}$on the ${ }^{182} \mathrm{~W}^{16} \mathrm{O}^{+}$ peak, and ${ }^{182} \mathrm{~W}^{18} \mathrm{O}^{+}$on the ${ }^{184} \mathrm{~W}^{16} \mathrm{O}^{+}$peak were found to be negligible, so no correction was applied. In addition, we carefully checked the mass region of interest for molecular interferences (Figure 3) resulting from complex combinations of major elements from the $\mathrm{SiC}$ matrix. Low count rates (e.g., $\sim 0.4$ counts $\mathrm{s}^{-1}$ at mass 198) were found in the mass region of interest when sputtering a "pure" synthetic SiC. Count rates at mass 198 sputtered from the KJB fraction are usually on the order of 20 counts $\mathrm{s}^{-1}$. Individual grains, on the other hand, had count rates between 1.5 and 8 counts $\mathrm{s}^{-1}$. The use of a small energy offset $(\sim 21-24 \mathrm{eV})$ has proved to be quite successful in suppressing complex molecular interferences without significantly compromising the intensity of the atomic species (Ávila et al. 2011). In this study, we confirmed this observation for the mass region of interest using a "pure" synthetic $\mathrm{SiC}$ reference material. As a result, $\mathrm{W}$ (and also $\mathrm{Hf}$ ) measurements in all individual $\mathrm{SiC}$ grains and 5 out of $11 \mathrm{KJB}$ spots (no. 07-11) were carried out on the SHRIMP-RG by combining high-mass resolution with energy filtering. Six KJB spots (no. 01-06) were analyzed without energy filtering. We found that all $\mathrm{KJB}$ spots have the same $\mathrm{W}$ isotopic composition within $2 \sigma$ errors. KJB spots 10 and 11 were analyzed only for ${ }^{179} \mathrm{Hf} /{ }^{180} \mathrm{Hf}$ ratios.

The acquisition time for each analysis was $\sim 6-7$ minutes. Because of the low $\mathrm{W}$ abundance in stardust $\mathrm{SiC}$ grains, the mass positions of $\mathrm{WO}^{+}$isotopic species could not be monitored during each individual analysis. Their mass positions were instead maintained from the previous measurement on the standard. We systematically bracketed three unknowns by a suite of standard reference materials (i.e., NIST-610 silicate glass and $\mathrm{SiC}$ ceramic). The shifts in peak positions monitored during the analytical sessions were found to be less than $0.002 \mathrm{amu}$ between consecutive standards. The secondary beam was aligned using the QQH monitor, allowing maximum transmission through the source slit. SHRIMP-RG was operated at a mass resolution of $m / \Delta m=7000$ (at $10 \%$ peak). The NIST-610 silicate glass and the $\mathrm{SiC}$ ceramic doped with heavy elements were used to monitor instrumental mass fractionation (IMF). The data were corrected for an IMF of $-12 \%$ amu ${ }^{-1}$ based on the ${ }^{186} \mathrm{~W} /{ }^{183} \mathrm{~W}$ ratio of 1.986 (Jacobsen 2005), and the mean of the ${ }^{186} \mathrm{~W} /{ }^{183} \mathrm{~W}$ measurements of the SiC standards. Mass interferences and background were monitored by periodically analyzing a "pure" synthetic $\mathrm{SiC}$ and the $\mathrm{Au}$ foil (i.e., the substrate on which the grains were deposited). The ${ }^{180} \mathrm{Hf} /{ }^{184} \mathrm{~W}$ ratios were normalized by applying the relative sensitivity factor determined by measuring the $\mathrm{SiC}$ ceramic doped with heavy elements.

The isotopic ratios obtained with SHRIMP-RG in singlecollection mode were calculated using Dodson's time- 

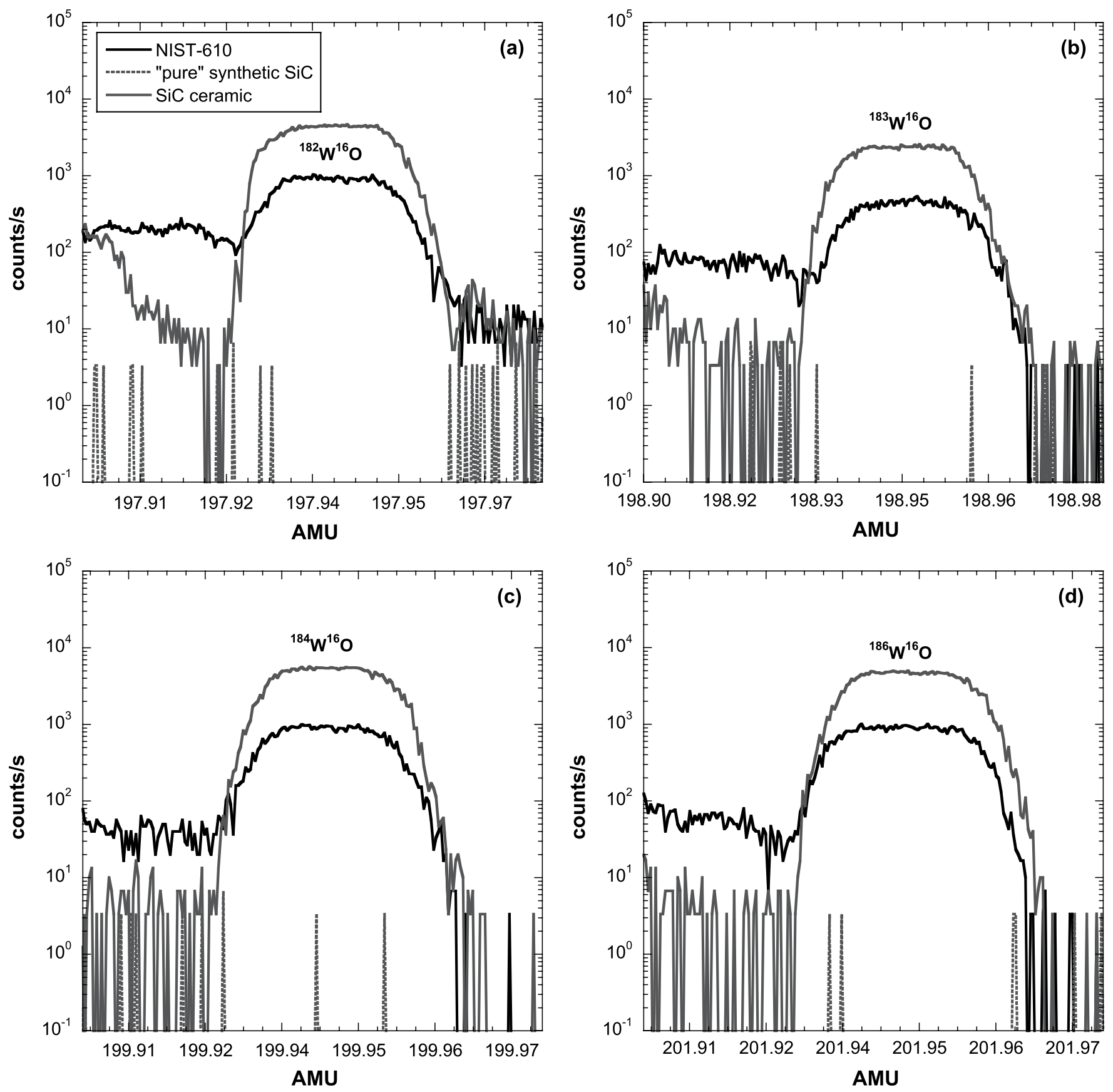

Figure 3. SHRIMP-RG mass scans of ${ }^{182} \mathrm{~W}^{16} \mathrm{O}^{+}(\mathrm{a}),{ }^{183} \mathrm{~W}^{16} \mathrm{O}^{+}(\mathrm{b}),{ }^{184} \mathrm{~W}^{16} \mathrm{O}^{+}(\mathrm{c})$, and ${ }^{186} \mathrm{~W}^{16} \mathrm{O}^{+}$(d), obtained in NIST-610 silicate glass, a "pure" synthetic SiC, and a $\mathrm{SiC}$ ceramic doped with heavy elements. Energy offset $=0 \mathrm{eV}$ and $m / \Delta m \sim 7000$ (at $10 \%$ peak).

interpolation algorithm (Dodson 1978), wherein the final isotopic ratios are calculated as means of $N-1$ interpolated ratios (where $N$ is the number of scans). To investigate the hypothesis that the isotopic measurements calculated using this approach could be affected by a systematic positive bias, we also calculated the isotopic ratios from the total counts as suggested by Huss et al. (2011) and Ogliore et al. (2011). We found slight differences $(<5 \%)$ in the final ratios, but all are well within the errors. The uncertainties of the $\mathrm{W}$ and $\mathrm{Hf}$ isotopic ratios measured on stardust $\mathrm{SiC}$ grains are dominated by counting statistics and were calculated from the standard deviation of the sampling distribution (i.e., standard error $=$ S.D. $/ \sqrt{n}$ ). Uncertainties related to the dispersion (i.e., standard deviation) of measurements on the standards during the analyti- cal session were calculated from repeated analysis of NIST-610 and $\mathrm{SiC}$ ceramic, and propagated into the uncertainty of each unknown.

\section{RESULTS}

Tungsten and hafnium isotopic compositions are reported in Table 2 . The quoted errors are $\pm 1 \sigma$. The reference $\mathrm{W}$ isotopic composition is taken from Jacobsen (2005). This composition is taken as a terrestrial composition that should also reflect the solar system composition (e.g., Lodders 2010), which is the case for most refractory elements. Isotope compositions are either reported directly or as delta $(\delta)$ values, defined as $\delta R=$ $\left[\left(R_{\text {measured }} / R_{\text {solar }}-1\right) \times 1000\right]$, where the measured isotopic 
Table 1

C-, N- and Si-isotopic Compositions of Stardust SiC Grains from the KJB and LS+LU Fractions Measured for W and Hf Isotopes

\begin{tabular}{|c|c|c|c|c|c|}
\hline Grain/Spot & $\begin{array}{l}\text { Size } \\
(\mu \mathrm{m})\end{array}$ & ${ }^{12} \mathrm{C} /{ }^{13} \mathrm{C} \pm 1 \sigma$ & ${ }^{14} \mathrm{~N} /{ }^{15} \mathrm{~N} \pm 1 \sigma$ & $\begin{array}{c}\delta^{29} \mathrm{Si} /{ }^{28} \mathrm{Si}^{\mathrm{a}} \pm 1 \sigma \\
(\% \circ)\end{array}$ & $\begin{array}{c}\delta^{30} \mathrm{Si} /{ }^{28} \mathrm{Si}^{\mathrm{a}} \pm 1 \sigma \\
(\% o)\end{array}$ \\
\hline \multicolumn{6}{|c|}{ KJB fraction (Murchison SiC-enriched sample) } \\
\hline KJB & 0.49 & $37.0 \pm 0.4$ & $521 \pm 60$ & $24.6 \pm 1.3$ & $37.8 \pm 3.4$ \\
\hline \multicolumn{6}{|c|}{ LS+LU fraction (Murchison single grains) } \\
\hline LU-34 & $7 \times 9$ & $54.3 \pm 0.4$ & $1889 \pm 13$ & $86.4 \pm 3.0$ & $73.2 \pm 3.4$ \\
\hline LU-36 & $23 \times 23$ & $49.1 \pm 0.4$ & $408 \pm 20$ & $37.9 \pm 2.5$ & $41.1 \pm 3.1$ \\
\hline LU-32 & $5 \times 13$ & $63.0 \pm 0.4$ & $1088 \pm 14$ & $55.3 \pm 2.5$ & $47.8 \pm 3.2$ \\
\hline LU-20 & $15 \times 26$ & $48.6 \pm 0.4$ & $935 \pm 22$ & $38.6 \pm 2.6$ & $45.0 \pm 3.5$ \\
\hline LU-41 & $13 \times 13$ & $48.4 \pm 0.3$ & $678 \pm 15$ & $43.3 \pm 3.4$ & $44.3 \pm 2.9$ \\
\hline
\end{tabular}

Notes. Isotopic ratios are reproduced from Amari et al. (2000) and Virag et al. (1992). Errors are $1 \sigma$.

${ }^{\mathrm{a}} \delta^{\mathrm{i}} \mathrm{Si} /{ }^{28} \mathrm{Si}(\% \circ)=\left[\left({ }^{\mathrm{i}} \mathrm{Si} /{ }^{28} \mathrm{Si}\right)_{\text {measured }} /\left({ }^{\mathrm{i}} \mathrm{Si} /{ }^{28} \mathrm{Si}\right)_{\text {solar }}-1\right] \times 10^{3}$.

ratios $\left(R_{\text {measured }}\right)$ are expressed as deviations from the reference terrestrial $(=$ solar $)$ isotopic composition $\left(R_{\text {solar }}\right)$ in parts per thousand $(\% \circ)$. Other interelement isotopic ratios are taken from Lodders (2010).

The weighted mean $\mathrm{W}$ isotopic ratios obtained for the SiC-enriched bulk sample (KJB fraction), based on nine measurements (Table 2 and Figure 4), are ${ }^{182} \mathrm{~W} /{ }^{184} \mathrm{~W}=0.809 \pm$ $0.014\left(\delta^{182} \mathrm{~W} /{ }^{184} \mathrm{~W}=-64 \% \circ \pm 17 \%\right.$ $),{ }^{183} \mathrm{~W} /{ }^{184} \mathrm{~W}=0.411$ $\pm 0.009\left(\delta^{183} \mathrm{~W} /{ }^{184} \mathrm{~W}=-120 \% \circ \pm 22 \%\right.$ o $)$, and ${ }^{186} \mathrm{~W} /{ }^{184} \mathrm{~W}=$ $0.944 \pm 0.024\left(\delta^{186} \mathrm{~W} /{ }^{184} \mathrm{~W}=18 \% \circ \pm 26 \%\right.$ o $)$. The KJB fraction shows a small deviation from solar composition in both ${ }^{182} \mathrm{~W} /$ ${ }^{184} \mathrm{~W}$ and ${ }^{183} \mathrm{~W} /{ }^{184} \mathrm{~W}$ ratios, with deficits in ${ }^{182} \mathrm{~W}$ and ${ }^{183} \mathrm{~W}$ with respect to ${ }^{184} \mathrm{~W}$. The ${ }^{186} \mathrm{~W} /{ }^{184} \mathrm{~W}$ ratio, however, shows no apparent deviation from the solar value, which raises the possibility of contamination with solar material. Although contamination is possible, we believe that it is unlikely since the other ratios (e.g., ${ }^{182} \mathrm{~W} /{ }^{184} \mathrm{~W}$ and ${ }^{183} \mathrm{~W} /{ }^{184} \mathrm{~W}$ ) are statistically anomalous.

Within the $\pm 1 \sigma$ uncertainties, the $\mathrm{W}$ isotopic ratios determined for two out of five single grains (LU-34 and LU-32) show no deviation from solar composition. On the other hand, two other grains (LU-36 and LU-41) exhibit deficits in ${ }^{182} \mathrm{~W}$, ${ }^{183} \mathrm{~W}$, and ${ }^{186} \mathrm{~W}$ with respect to ${ }^{184} \mathrm{~W}$. Grain LU-20 shows a deviation from the solar value only in the ${ }^{186} \mathrm{~W} /{ }^{184} \mathrm{~W}$ ratio, with both ${ }^{182} \mathrm{~W} /{ }^{184} \mathrm{~W}$ and ${ }^{183} \mathrm{~W} /{ }^{184} \mathrm{~W}$ ratios showing no apparent deviation from solar within errors. Only grain LU-41 has deviations from the solar isotopic ratios that are larger than $2 \sigma$. No obvious correlations between $\mathrm{W}$ isotopic compositions and $\mathrm{C}-, \mathrm{N}-$, and $\mathrm{Si}$-isotopic compositions were found.

Additionally, we provide information on the ${ }^{179} \mathrm{Hf} /{ }^{180} \mathrm{Hf}$ ratios (Table 2). The weighted mean ${ }^{179} \mathrm{Hf} /{ }^{180} \mathrm{Hf}$ of the $\mathrm{SiC}$ enriched bulk sample (KJB fraction), based on three measurements, is $0.326 \pm 0.033\left(\delta^{179} \mathrm{Hf} /{ }^{180} \mathrm{Hf}=-160 \%\right.$ $\pm 85 \%$ o $)$. This value deviates only slightly from that reported for the solar system $\left({ }^{179} \mathrm{Hf} /{ }^{180} \mathrm{Hf}=0.388\right.$; Lodders 2010). Three out of five investigated single grains had sufficiently high Hf concentrations for isotopic analysis. Two of them show no apparent deviation from the solar value (within $1 \sigma$ error). Grain LU-41, which has the largest deficit in ${ }^{182} \mathrm{~W},{ }^{183} \mathrm{~W}$, and ${ }^{186} \mathrm{~W}$ with respect to ${ }^{184} \mathrm{~W}$, also has a significant deficit in ${ }^{179} \mathrm{Hf}$ with respect to ${ }^{180} \mathrm{Hf}\left(\delta^{179} \mathrm{Hf} /{ }^{180} \mathrm{Hf}=-637 \% \circ \pm 121 \%\right.$ o $)$. All single grains and KJB spot analyses show lower ${ }^{180} \mathrm{Hf} /{ }^{184} \mathrm{~W}$ ratios than current predictions from $s$-process nucleosynthesis calculations (see Section 4).

We shall now consider some factors that may affect the present determinations. First, there is a potential problem of contamination with foreign materials and minerals, originating either from the meteorite itself or as a result of sample preparation. Tungsten, unfortunately, may be a contaminant from the sample preparation. Sodium polytungstate was used for density separation of graphite from $\mathrm{SiC}$ (Amari et al. 1994). The density separation was followed by several washing procedures in order to remove any contaminants. Based on the behavior of the isotopic ratios as a function of acquisition time, there is no evidence from our analyses to suggest that the $\mathrm{W}$ isotopic compositions are not intrinsic to the grains or result from surface contamination. Furthermore, we did not detect $\mathrm{W}$ in the Au-foil, onto which both the KJB and LS+LU fractions had been deposited. Nonetheless, three out of five single grains show no apparent deviation from the solar isotopic ratios (for both $\mathrm{W}$ and $\mathrm{Hf}$ ), and contamination with solar material cannot be completely ruled out. Kashiv (2004) found very high W abundance in stardust $\mathrm{SiC}$ grains (W enrichment factor, relative to solar $\mathrm{W} / \mathrm{Si}$ ratio, of 300-8700), much higher than expected based on AGB stellar models and thermodynamic condensation calculations. In contrast to Kashiv (2004), our W concentration measurements are semi-quantitative only. Nevertheless, we found for the KJB fraction a $\mathrm{W}$ enrichment factor $\sim 10$, and for the single grains from the LS+LU fraction an enrichment factor $<3$, which are much lower than the values found by Kashiv (2004). This author also suggested that $\mathrm{W}$ contamination could be from heavy metal alloy holders used during analytical procedures. Contamination from the Murchison meteorite is another possibility. In order to minimize any residual surface contamination, all spots and grains analyzed were initially rastered across an area slightly larger than the analytical pit by the $\mathrm{O}_{2}^{-}$primary beam for $\sim 60 \mathrm{~s}$ before data acquisition.

Another complication encountered during ion microprobe analysis of $\mathrm{SiC}$ grains is the lack of suitable $\mathrm{SiC}$ standards. The accuracy and precision of ion microprobe measurements is known to be highly dependent on the availability of suitable standards because of the variability of ion emission produced by sputtering of solid geological and cosmochemical materials. Standards that are compositionally and structurally similar to the analytical target, i.e., matrix-matched, are therefore highly desirable. Furthermore, assessment of molecular interferences produced by combinations of isotopes of the major elements is particularly important for the analysis of trace elements where even weak molecular interferences may significantly contribute to the mass peak being measured. Naturally occurring (except for stardust $\mathrm{SiC}$ grains) and synthetic $\mathrm{SiC}$ samples contain very low abundances of trace elements and are therefore not suitable as standard materials. While the synthesis of large doped 
Table 2

Tungsten and Hafnium Isotopic Ratios Determined in Stardust SiC Grains

\begin{tabular}{|c|c|c|c|c|c|c|c|c|c|}
\hline Spot/Grain & ${ }^{182} \mathrm{~W} /{ }^{184} \mathrm{~W} \pm 1 \sigma$ & $\begin{array}{c}\delta^{182} \mathrm{~W} /{ }^{184} \mathrm{~W}^{\mathrm{b}} \pm 1 \sigma \\
(\% \circ)\end{array}$ & ${ }^{183} \mathrm{~W} /{ }^{184} \mathrm{~W} \pm 1 \sigma$ & $\begin{array}{c}\delta^{183} \mathrm{~W} /{ }^{184} \mathrm{~W}^{\mathrm{b}} \pm 1 \sigma \\
(\% o)\end{array}$ & ${ }^{186} \mathrm{~W} /{ }^{184} \mathrm{~W} \pm 1 \sigma$ & $\begin{array}{c}\delta^{186} \mathrm{~W} /{ }^{184} \mathrm{~W}^{\mathrm{b}} \pm 1 \sigma \\
(\% \circ)\end{array}$ & ${ }^{179} \mathrm{Hf} /{ }^{180} \mathrm{Hf} \pm 1 \sigma$ & $\begin{array}{c}\delta^{179} \mathrm{Hf} /{ }^{180} \mathrm{Hf}^{\mathrm{c}} \pm 1 \sigma \\
(\% \circ)\end{array}$ & ${ }^{180} \mathrm{Hf} /{ }^{184} \mathrm{~W}^{\mathrm{d}} \pm 1 \sigma$ \\
\hline Terrestrial $^{\mathrm{a}}$ & 0.865 & & 0.467 & & 0.928 & & 0.388 & & 1.302 \\
\hline \multicolumn{10}{|c|}{ KJB fraction (Murchison SiC-enriched sample) } \\
\hline KJB-01 & $0.725 \pm 0.054$ & $-162 \pm 63$ & $0.395 \pm 0.027$ & $-154 \pm 57$ & $0.755 \pm 0.077$ & $-186 \pm 83$ & n.a. & & n.a. \\
\hline KJB-02 & $0.824 \pm 0.044$ & $-48 \pm 51$ & $0.434 \pm 0.050$ & $-70 \pm 108$ & $0.858 \pm 0.052$ & $-76 \pm 56$ & n.a. & & $0.095 \pm 0.013$ \\
\hline KJB-03 & $0.817 \pm 0.024$ & $-56 \pm 28$ & $0.399 \pm 0.015$ & $-147 \pm 31$ & $0.985 \pm 0.029$ & $62 \pm 31$ & n.a. & & $0.046 \pm 0.002$ \\
\hline KJB-04 & $0.752 \pm 0.045$ & $-130 \pm 52$ & $0.395 \pm 0.029$ & $-154 \pm 62$ & $0.904 \pm 0.056$ & $-25 \pm 60$ & n.a. & & $0.041 \pm 0.004$ \\
\hline KJB-05 & $0.825 \pm 0.043$ & $-46 \pm 50$ & $0.394 \pm 0.026$ & $-157 \pm 57$ & $0.954 \pm 0.054$ & $28 \pm 58$ & n.a. & & $0.097 \pm 0.015$ \\
\hline KJB-06 & $0.838 \pm 0.056$ & $-31 \pm 64$ & $0.461 \pm 0.037$ & $-12 \pm 78$ & $0.963 \pm 0.044$ & $39 \pm 47$ & n.a. & & $0.059 \pm 0.009$ \\
\hline KJB-07 & $0.845 \pm 0.055$ & $-23 \pm 63$ & $0.454 \pm 0.036$ & $-28 \pm 76$ & $0.995 \pm 0.065$ & $72 \pm 70$ & n.a. & & $0.057 \pm 0.012$ \\
\hline KJB-08 & $0.829 \pm 0.058$ & $-42 \pm 67$ & $0.451 \pm 0.038$ & $-35 \pm 80$ & $0.996 \pm 0.068$ & $74 \pm 72$ & n.a. & & $0.068 \pm 0.007$ \\
\hline KJB-09 & $0.817 \pm 0.056$ & $-55 \pm 64$ & $0.436 \pm 0.036$ & $-66 \pm 77$ & $0.902 \pm 0.064$ & $-28 \pm 69$ & $0.338 \pm 0.060$ & $-130 \pm 155$ & $0.069 \pm 0.007$ \\
\hline KJB-10 & n.a. & & n.a. & & n.a. & & $0.318 \pm 0.059$ & $-181 \pm 153$ & n.a. \\
\hline KJB-11 & n.a. & & n.a. & & n.a. & & $0.322 \pm 0.056$ & $-170 \pm 144$ & n.a. \\
\hline Weighted average & $0.809 \pm 0.014$ & $-64 \pm 17$ & $0.411 \pm 0.009$ & $-120 \pm 22$ & $0.944 \pm 0.024$ & $18 \pm 26$ & $0.326 \pm 0.033$ & $-160 \pm 85$ & $0.050 \pm 0.005$ \\
\hline \multicolumn{10}{|c|}{ LS+LU fraction (Murchison single grains) } \\
\hline LU-34 & $0.866 \pm 0.067$ & $1 \pm 77$ & $0.485 \pm 0.044$ & $38 \pm 95$ & $0.887 \pm 0.069$ & $-44 \pm 75$ & $0.475 \pm 0.076$ & $223 \pm 195$ & $0.194 \pm 0.035$ \\
\hline LU-36 & $0.722 \pm 0.070$ & $-165 \pm 80$ & $0.384 \pm 0.061$ & $-177 \pm 130$ & $0.802 \pm 0.091$ & $-136 \pm 98$ & b.d.l. & & $0.070 \pm 0.009$ \\
\hline LU-32 & $0.848 \pm 0.072$ & $-20 \pm 83$ & $0.463 \pm 0.048$ & $-8 \pm 102$ & $0.870 \pm 0.076$ & $-62 \pm 81$ & $0.437 \pm 0.078$ & $126 \pm 200$ & $0.203 \pm 0.016$ \\
\hline LU-20 & $0.802 \pm 0.071$ & $-73 \pm 83$ & $0.439 \pm 0.042$ & $-61 \pm 89$ & $0.769 \pm 0.070$ & $-171 \pm 76$ & b.d.l. & & $0.111 \pm 0.030$ \\
\hline LU-41 & $0.648 \pm 0.056$ & $-250 \pm 65$ & $0.304 \pm 0.034$ & $-349 \pm 73$ & $0.639 \pm 0.058$ & $-312 \pm 63$ & $0.141 \pm 0.047$ & $-637 \pm 121$ & $0.276 \pm 0.047$ \\
\hline
\end{tabular}

Notes. Errors are $1 \sigma$. n.a. $=$ not analyzed; b.d.l. = below detection limit.

${ }^{\text {a } 182} \mathrm{~W} /{ }^{184} \mathrm{~W},{ }^{183} \mathrm{~W} /{ }^{184} \mathrm{~W}$, and ${ }^{186} \mathrm{~W} /{ }^{184} \mathrm{~W}$ from Jacobsen (2005). ${ }^{179} \mathrm{Hf} /{ }^{180} \mathrm{Hf}$ and ${ }^{180} \mathrm{Hf} /{ }^{184} \mathrm{~W}$ from Lodders (2010).

$\left.{ }^{\mathrm{b}} \delta^{\mathrm{i}} \mathrm{W} /{ }^{184} \mathrm{~W}(\% \circ)=\left[{ }^{\mathrm{i}} \mathrm{W} /{ }^{184} \mathrm{~W}\right)_{\text {measured }} /\left({ }^{\mathrm{i}} \mathrm{W} /{ }^{184} \mathrm{~W}\right)_{\text {solar }}-1\right] \times 10^{3}$

c $\delta^{179} \mathrm{Hf} /{ }^{180} \mathrm{Hf}(\%)=\left[\left({ }^{179} \mathrm{Hf} /{ }^{180} \mathrm{Hf}\right)_{\text {measured }} /\left({ }^{179} \mathrm{Hf} /{ }^{180} \mathrm{Hf}\right)_{\text {solar }}-1\right] \times 10^{3}$.

${ }^{\mathrm{d}}$ Corrected with relative sensitivity factor determined on the $\mathrm{SiC}$ ceramic. 


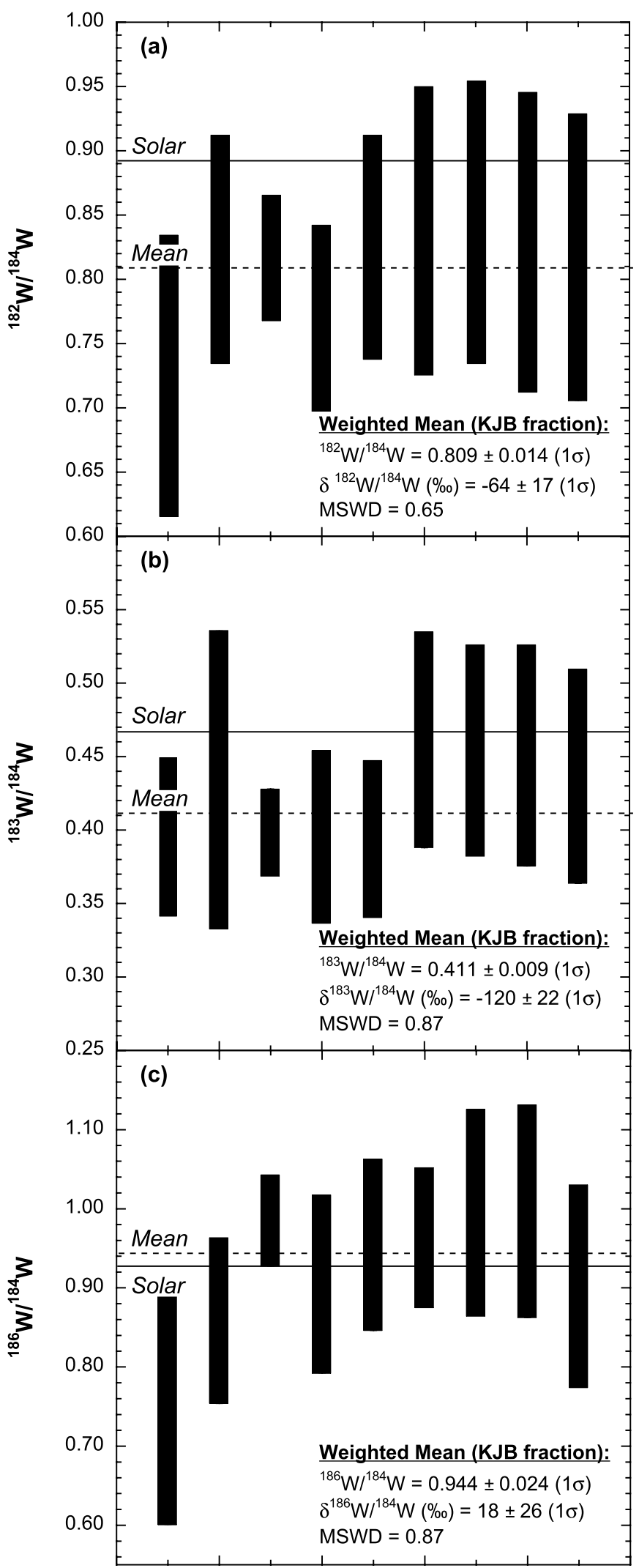

Figure 4. Tungsten isotopic compositions determined for the SiC-enriched sample (KJB fraction). Each bar represents an individual measurement. Bold and dashed lines indicate solar ratios (as inferred from terrestrial composition; Jacobsen 2005) and SiC weighted mean ratios, respectively. Box heights are $2 \sigma$. Weighted means are also reported as deviations ( $\delta$-values) from the reference terrestrial (= solar) isotopic composition in parts per thousand (\%o). MSWD is the mean square weighted deviation.
$\mathrm{SiC}$ crystals in the laboratory is extremely difficult, SiC-based ceramics sintered with desirable amounts of trace elements are relatively easy to produce. In order to determine elemental yields and identify possible molecular interferences in the mass region of $\mathrm{W}$ isotopes, we prepared a $\mathrm{SiC}$ ceramic doped with several trace elements at nominal concentrations between 1 and $3000 \mathrm{ppm}$. The SiC ceramic has proved to be a useful standard for elemental and isotopic measurements in stardust SiC grains, especially concerning identification of molecular interferences.

\section{W AND Hf CONDENSATION INTO SiC GRAINS FROM AGB STARS}

The ${ }^{180} \mathrm{Hf} /{ }^{184} \mathrm{~W}$ ratios obtained here for stardust $\mathrm{SiC}$ grains (0.041-0.276) are lower than $s$-process model predictions for the envelope compositions of carbon-rich, low-mass AGB stars with close-to-solar metallicity $\left(\sim 1.42^{9}\right.$; solar system $\sim 1.30$; Lodders 2010). Clearly, it is not possible to attribute the observed range to nucleosynthetic effects or to contamination with solar material. Hence, we turn to discussing the condensation behavior of $\mathrm{W}$ and $\mathrm{Hf}$ to see if this could provide an explanation. According to Lodders \& Fegley (1995), under the same conditions of pressure and $\mathrm{C} / \mathrm{O}$ ratio, $\mathrm{W}$ condenses as $\mathrm{WC}$ at a temperature $\sim 100 \mathrm{~K}$ higher than HfC. Both WC and HfC are more refractory than $\mathrm{SiC}$. Thus, one would expect both elements to fully condense into $\mathrm{SiC}$, or at least in the same proportion. Based on our data, this appears not to be the case. One way to explain small fractionations between $\mathrm{Hf}$ and $\mathrm{W}$ is by having $\mathrm{W}$ condense as a metal instead of WC. This can occur if condensation takes place at pressures higher than $10^{-4}$ bars and if $\mathrm{W}$ in the circumstellar envelope reaches a concentration of at least 10 times the abundance found in the solar system (Lodders \& Fegley 1995). We note that the $\mathrm{W}$ enrichment factor determined for the KJB fraction is $\sim 10$, which is comparable to the results obtained for other heavy $s$-process elements. However, the single $\mathrm{SiC}$ grains from the $\mathrm{LS}+\mathrm{LU}$ fraction show an enrichment factor $<3$. Large fractionation between elements present in $\mathrm{SiC}$ relative to the source composition had been previously observed (e.g., $\mathrm{Al} / \mathrm{Mg}, \mathrm{Sr} / \mathrm{Ba}$ : Amari et al. 1995; Ni/Fe: Marhas et al. 2008), but there is still no satisfactory explanation for these elemental fractionations.

\section{THE $s$-PROCESS PATH IN THE W MASS REGION}

The $s$-process path in the mass region around $\mathrm{W}$ is shown in Figure 1. The $s$-process takes place in the deep He-rich layers of low- and intermediate-mass stars $\left(M \sim 0.8-8 M_{\odot}\right)$ during their AGB phase of evolution (e.g., Gallino et al. 1998; Busso et al. 1999; Herwig 2005; Zinner et al. 2006; Cristallo et al. 2009). In low-mass AGB stars of close-to-solar metallicity, believed to be the site of origin of mainstream $\mathrm{SiC}$ grains, the ${ }^{13} \mathrm{C}(\alpha, n){ }^{16} \mathrm{O}$ reaction is the main neutron source responsible for the production of the bulk of the s-process AGB yields. It usually operates under radiative conditions at relatively low temperatures $\left(T \sim 0.9 \times 10^{8} \mathrm{~K}\right.$, corresponding to a thermal energy of $k T \sim 8 \mathrm{keV}$ ), during the interval between episodic He burning (thermal pulses), and results in low neutron densities $\left(10^{6}-10^{7}\right.$ neutrons $\mathrm{cm}^{-3}$; Straniero et al. 1995). A second neutron source, the ${ }^{22} \mathrm{Ne}(\alpha, n)^{25} \mathrm{Mg}$ reaction, is marginally

\footnotetext{
9 Arithmetic average composition found in the stellar envelope after the last thermal pulse with third dredge-up of the $1.5,2,2.5$, and $3 M_{\odot}$ FRANEC AGB models at $\boldsymbol{Z}=0.01,0.014$, and 0.02 (FRUITY database,

http://fruity.oa-teramo.inaf.it:8080/modelli.pl; Cristallo et al. 2011) and 1.25, $1.8,3$, and $4 M_{\odot}$ MONASH AGB models at $\boldsymbol{Z}=0.01$ and 0.02 .
} 
activated during thermal pulses when the maximum temperature at the bottom of the He-burning shell reaches $T \sim 3 \times 10^{8} \mathrm{~K}$ $\left(k T \sim 23 \mathrm{keV}\right.$ ), resulting in high neutron densities (up to $\sim 10^{10}$ neutrons $\mathrm{cm}^{-3}$ in AGB stars of initial mass $\left.1.5-3 M_{\odot}\right)$. Although neutron captures during these short episodes account only for a few percent of the total exposure, they are essential for adjusting the abundance patterns of the $s$-process branchings.

The production of $\mathrm{W}$ in the He-intershell during interpulse and thermal pulse phases is strongly affected by branchings at ${ }^{181} \mathrm{Hf},{ }^{182} \mathrm{Hf},{ }^{182} \mathrm{Ta},{ }^{183} \mathrm{Ta}$, and ${ }^{185} \mathrm{~W}$ (see Figure 1). The competition between neutron capture and $\beta$-decay at these branching points can be expressed by a branching factor $\left(f_{n}\right)$, calculated from $f_{n}=\lambda_{n} /\left(\lambda_{n}+\lambda_{\beta}\right)$, where $\lambda_{n}=N_{n} \nu_{T}\langle\sigma\rangle$ and $\lambda_{\beta}=\ln 2 / t_{1 / 2}$ are the neutron-capture rate and the $\beta$-decay rate, respectively. Here, $N_{n}, v_{T},\langle\sigma\rangle$, and $t_{1 / 2}$ are the neutron density, the thermal velocity, the Maxwellian-averaged $(n, \gamma)$ cross section, and the half-life, respectively. At the typical conditions of operation of the ${ }^{13} \mathrm{C}(\alpha, n){ }^{16} \mathrm{O}$ neutron source $\left(T \sim 0.9 \times 10^{8} \mathrm{~K}, N_{n} \sim 10^{6}\right.$ to $10^{7}$ neutrons $\left.\mathrm{cm}^{-3}\right)$, the ${ }^{181} \mathrm{Hf},{ }^{182} \mathrm{Ta}$, and ${ }^{183} \mathrm{Ta}$ branching factors are very small $(<0.2 \%$, $1.5 \%$, and $<0.1 \%$, respectively, see Figure 5(a)), indicating that ${ }^{181} \mathrm{Hf},{ }^{182} \mathrm{Ta}$, and ${ }^{183} \mathrm{Ta}$ will $\beta$-decay to ${ }^{181} \mathrm{Ta},{ }^{182} \mathrm{~W}$, and ${ }^{183} \mathrm{~W}$, respectively, rather than capture a neutron. Hence, the $s$-process flow will create ${ }^{182} \mathrm{~W},{ }^{183} \mathrm{~W}$, and ${ }^{184} \mathrm{~W}$ via ${ }^{181} \mathrm{Hf}(\beta, \nu){ }^{181} \mathrm{Ta}(n, \gamma)$ ${ }^{182} \mathrm{Ta}(\beta, \nu){ }^{182} \mathrm{~W}(n, \gamma){ }^{183} \mathrm{~W}(n, \gamma){ }^{184} \mathrm{~W}$. The branching point at ${ }^{185} \mathrm{~W}$ also shows a small probability $(\sim 1 \%)$ toward ${ }^{186} \mathrm{~W}$ (Figure $5(\mathrm{a})$ ), so that no ${ }^{186} \mathrm{~W}$ will be produced during the activation of the ${ }^{13} \mathrm{C}$ neutron source.

At higher temperatures and neutron density conditions typically found during the AGB thermal pulses ( $T$ up to $3 \times 10^{8} \mathrm{~K}$, $N_{n}$ up to $\sim 10^{10}$ neutrons $\mathrm{cm}^{-3}$ ), the $s$-process production of ${ }^{182} \mathrm{~W},{ }^{183} \mathrm{~W}$, and ${ }^{186} \mathrm{~W}$ is different. Under these conditions, the branching factor at ${ }^{181} \mathrm{Hf}$ is $\sim 10 \%$ at $N_{n}=10^{10}$ neutrons $\mathrm{cm}^{-3}$ (Figure 5(b)), marginally feeding ${ }^{182} \mathrm{Hf}$. The laboratory halflife of ${ }^{182} \mathrm{Hf}$ is $8.9 \times 10^{6} \mathrm{yr}$ (Vockenhuber et al. 2004), but at $T \sim 8 \mathrm{keV}$ and $23 \mathrm{keV}$ (and electron density $\sim 5 \times 10^{26} \mathrm{~cm}^{-3}$ ) it drops to $\sim 41 \times 10^{3} \mathrm{yr}$ and $12 \mathrm{yr}$, respectively, as a result of increased thermal populations of low-lying nuclear states (Takahashi \& Yokoi 1987). Despite the enhancement observed in the ${ }^{182} \mathrm{Hf} \beta$-decay rate at stellar conditions, its half-life is still sufficiently long to allow neutron captures to occur. Therefore, ${ }^{182} \mathrm{Hf}$ captures a neutron rather than decay during both interpulse and thermal pulse phases. Provided that the ${ }^{181} \mathrm{Hf}$ branching point is open, the $s$-process flow will proceed via the sequence ${ }^{181} \operatorname{Hf}(n, \gamma){ }^{182} \operatorname{Hf}(n, \gamma){ }^{183} \operatorname{Hf}(\beta, \nu){ }^{183} \mathrm{Ta}(\beta, \nu){ }^{183} \mathrm{~W}$, consequently bypassing ${ }^{182} \mathrm{~W}$, as well as ${ }^{183} \mathrm{~W}$ if the branching point at ${ }^{183} \mathrm{Ta}$ is also activated (see below). After the neutron flow ceases, ${ }^{182} \mathrm{~W}$ is marginally produced by the decay of ${ }^{182} \mathrm{Hf}$. Low-mass AGB stars yield ${ }^{182} \mathrm{Hf} /{ }^{180} \mathrm{Hf}$ ratios of $\sim 1.79 \times$ $10^{-2}$, which means that the effect of the ${ }^{181} \mathrm{Hf}$ branching point is marginal. Only a very small shift of $\sim 1 \%-3 \%$ in the ${ }^{182} \mathrm{~W} /{ }^{184} \mathrm{~W}$ ratio due to ${ }^{182} \mathrm{Hf}$ decay is expected if both $\mathrm{Hf}$ and $\mathrm{W}$ fully condense into $\mathrm{SiC}$. This shift is likely undetectable given the low ${ }^{180} \mathrm{Hf} /{ }^{184} \mathrm{~W}$ ratios determined here, which imply a very low $s$-process abundance of ${ }^{182} \mathrm{Hf}$ in the studied SiC grains. Hence, the observable radiogenic contribution of ${ }^{182} \mathrm{Hf}$ to its daughter ${ }^{182} \mathrm{~W}$ in the grains would be much less than $1 \%$ and thus too difficult to observe.

The $s$-process flow that goes through ${ }^{181} \mathrm{Hf}(\beta, \nu){ }^{181} \mathrm{Ta}(n, \gamma)$ ${ }^{182} \mathrm{Ta}$ encounters two other branching points at ${ }^{182} \mathrm{Ta}$ and ${ }^{183} \mathrm{Ta}$. The ${ }^{182} \mathrm{Ta}$ branching point has a temperaturedependent $\beta$-decay rate and a branching factor $>80 \%$ (Figure 5(b)) during the high neutron density produced by the

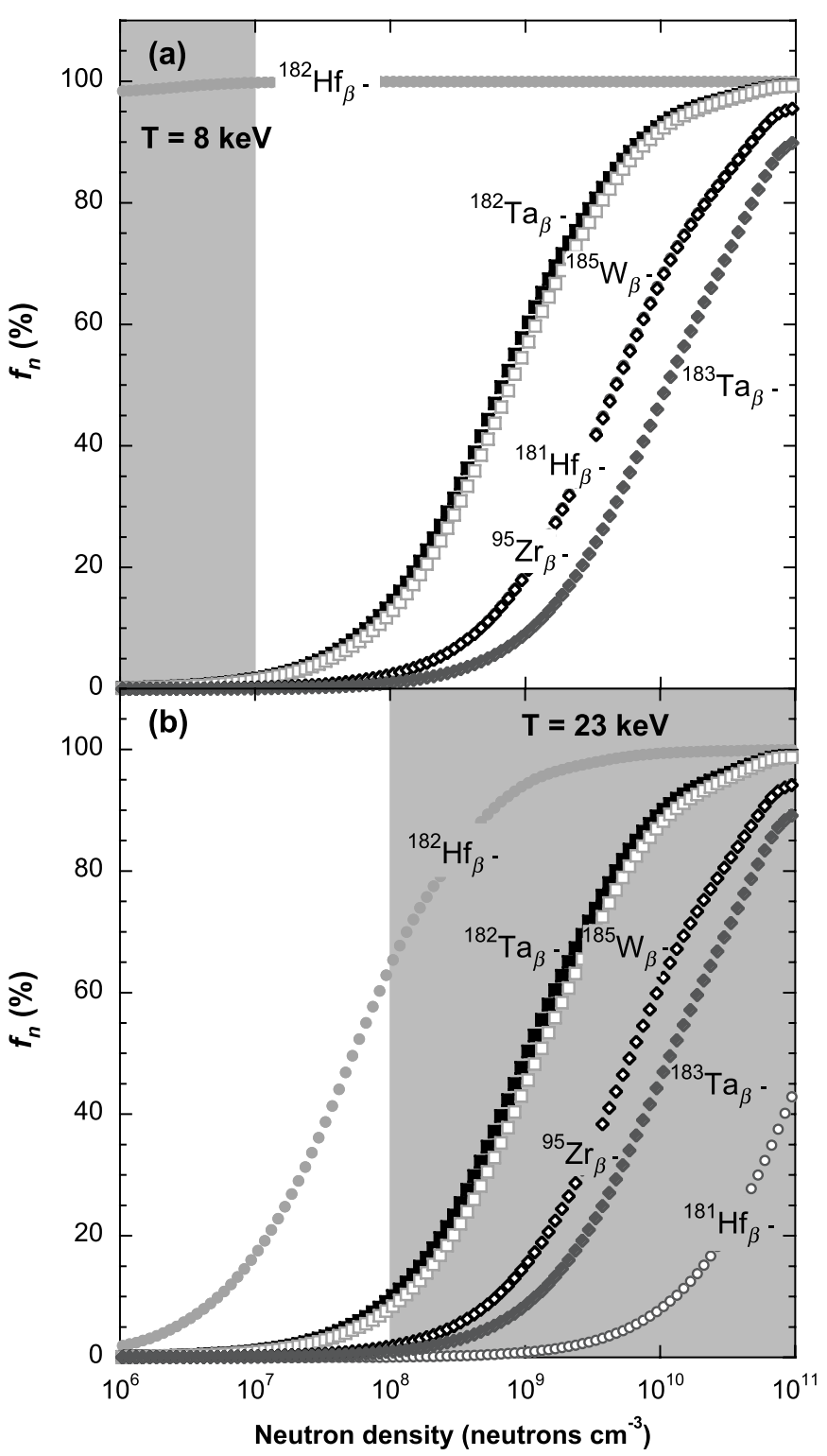

Figure 5. Branching factor $\left(f_{n}\right)$ of ${ }^{181} \mathrm{Hf},{ }^{182} \mathrm{Hf},{ }^{182} \mathrm{Ta},{ }^{183} \mathrm{Ta},{ }^{185} \mathrm{~W}$, and ${ }^{95} \mathrm{Zr}$ at $k T=8 \mathrm{keV}$ (a) and $23 \mathrm{keV}$ (b) as a function of neutron density. We used the $\beta$-decay rates reported by Takahashi \& Yokoi (1987) and the latest accepted neutron-capture rates from the KADoNiS Database (http://www.kadonis.org/; Dillmann et al. 2006), except for the ${ }^{183} \mathrm{Ta}$, where the neutron-capture rate from JINA REACLIB database (http://groups.nscl.msu.edu/jina/reaclib/db/; Cyburt et al. 2010) was used. All values were calculated for an electron density of 5 $\times 10^{26} \mathrm{~cm}^{-3}$. The branching factor (\%) depends on the neutron-capture and $\beta^{-}$decay rates and indicates the probability that an unstable isotope will capture a neutron rather than decay. The gray area in panels (a) and (b) corresponds to the conditions typically found during interpulse and thermal pulse phases, respectively, in low-mass AGB stars.

${ }^{22} \mathrm{Ne}$ source, and may cause the $s$-process flow to partially bypass ${ }^{182} \mathrm{~W}$. The branching factor at ${ }^{183} \mathrm{Ta}$ is $\sim 50 \%$ at $T \sim 3 \times$ $10^{8} \mathrm{~K}$ and $N_{n}=10^{10}$ neutrons $\mathrm{cm}^{-3}$ (Figure $5(\mathrm{~b})$ ), causing the $s$-process flow to partially bypass ${ }^{183} \mathrm{~W}$. The branching factor at ${ }^{185} \mathrm{~W}$ is enhanced similarly to the ${ }^{182} \mathrm{Ta}$ branching factor during thermal pulses (Figure $5(\mathrm{~b})$ ), feeding ${ }^{186} \mathrm{~W}$, which results in a smaller $s$-process contribution to both ${ }^{186} \mathrm{Os}$ and ${ }^{187} \mathrm{Os}$, due to the presence of the long-living nucleus ${ }^{187} \mathrm{Re}$. The ${ }^{186} \mathrm{~W} /{ }^{184} \mathrm{~W}$ ratios reported here suggest activation of the branching point at ${ }^{185} \mathrm{~W}$. This result is, in principle, in disagreement with ${ }^{96} \mathrm{Zr}$ depletions observed in $\mathrm{SiC}$ grains that indicate that the 
${ }^{22} \mathrm{Ne}$ neutron source was weak in the parent stars of the grains (Nicolussi et al. 1997; Lugaro et al. 2003). However, at conditions of $T \sim 23 \mathrm{keV}$ and $N_{n}>5 \times 10^{8}$ neutrons $\mathrm{cm}^{-3}$, the branching point at ${ }^{95} \mathrm{Zr}$ shows a smaller branching factor than ${ }^{185} \mathrm{~W}$ (Figure $5(\mathrm{~b})$ ). Therefore, at the same conditions, the ${ }^{185} \mathrm{~W}(n, \gamma){ }^{186} \mathrm{~W}$ reaction shows a higher probability to occur than the ${ }^{95} \mathrm{Zr}(n, \gamma){ }^{96} \mathrm{Zr}$ reaction. Information about the $\mathrm{Zr}$ isotopic compositions in the same grains measured for $\mathrm{W}$ would help to better constrain the physical conditions of the $s$-process in the parent stars of the grains.

From Figure 1, it can be seen that there is only one path that leads from ${ }^{179} \mathrm{Hf}$ to ${ }^{180} \mathrm{Hf},{ }^{10}$ whose relative abundances are not affected by any branching point. If a steady flow is achieved along the $s$-process path, the local equilibrium approximation applies $\langle\sigma\rangle_{(A)} N_{S(A)} \sim\langle\sigma\rangle_{(A-1)} N_{S(A-1)}$, where $\langle\sigma\rangle_{(A)}$ is the Maxwellian averaged $(n, \gamma)$ cross section of the isotope A, and $N_{s}$ is its $s$-process abundance (Clayton 1983). From this simple formulation, we can infer that the ratio of the $s$-process contributions to ${ }^{179} \mathrm{Hf}$ and ${ }^{180} \mathrm{Hf}$ are approximately equal to the inverse ratios of their neutron-capture cross sections. At thermal energies of $\sim 8$ and $23 \mathrm{keV}$, the inverse ratio of the ${ }^{179} \mathrm{Hf} /$ ${ }^{180} \mathrm{Hf}$ neutron-capture cross sections yields $\langle\sigma\rangle_{\left({ }^{180} \mathrm{Hf}\right)} /\langle\sigma\rangle_{\left({ }^{179} \mathrm{Hf}\right)}$ $=0.150$ and 0.175 , respectively, with an uncertainty of $\sim 1.5 \%$ (Dillmann et al. 2006). The grain showing the most anomalous $\mathrm{W}$ and $\mathrm{Hf}$ isotopic ratios, LU-41, has ${ }^{179} \mathrm{Hf} /{ }^{180} \mathrm{Hf}=0.141 \pm$ 0.047 , in very good agreement with the ratio expected from the values of neutron-capture cross sections, thus indicating an almost pure $s$-process signature in this grain.

\section{DISCUSSION}

Figures 6 and 7 show the $\mathrm{W}$ and $\mathrm{Hf}$ isotopic ratios of the stardust $\mathrm{SiC}$ grains together with $s$-process model predictions for the envelope compositions of low-mass AGB stars. These predictions represent the mixing between two components, one close to the solar composition, representing the initial composition of the stellar envelope (in stardust studies this is traditionally referred to as the $N$-component), the other with isotopic characteristics close to those predicted for pure $s$-process (the $G$-component; e.g., Zinner et al. 1991; Nicolussi et al. 1997; Savina et al. 2004). The magnitude of the departure from the $N$-component toward the $G$-component depends on the efficiency and the number of mixing episodes, also known as third dredge-ups (TDUs), which occur when the convective envelope penetrates into the He intershell zone. The TDU causes newly synthesized ${ }^{12} \mathrm{C}$ and $s$-processed material to be mixed into the convective envelope of the star (e.g., Lugaro et al. 2003).

Two different sets of models (FRANEC, Cristallo et al. 2009, 2011; MONASH, Karakas 2010, Karakas et al. 2010) with a range of stellar masses $\left(1.25-4 M_{\odot}\right)$ and metallicities $(Z=0.01$, 0.014 , and 0.02) were investigated. Of relevance here, in the FRANEC code the neutron-capture cross sections for the $\mathrm{W}$ isotopes are taken from Bao et al. (2000), and correspond to the values published by Macklin et al. (1983) for ${ }^{182} \mathrm{~W},{ }^{183} \mathrm{~W}$, ${ }^{184} \mathrm{~W}$, and ${ }^{186} \mathrm{~W}$, and the theoretical value adopted by Bao et al. (2000) for ${ }^{185} \mathrm{~W}$. In the MONASH code, the neutron-capture

\footnotetext{
10 A branching point at ${ }^{179} \mathrm{Hf}$ may be activated because this stable nucleus becomes unstable in stellar conditions. This effect is insignificant in our context, as the $\beta$-decay half-life of ${ }^{179} \mathrm{Hf}$ is $\sim 30 \mathrm{yr}$ at $\boldsymbol{T}=3 \times 10^{8} \mathrm{~K}$, however, in some conditions it may lead to the production of ${ }^{180} \mathrm{Ta}$ (actually an isomeric state, ${ }^{180} \mathrm{Ta}^{\mathrm{m}}$ ), the least abundant nucleus in the solar system (e.g., Käppeler et al. 2004). A weak s-process branching may also be activated via neutron captures on ${ }^{179} \mathrm{Hf}$, feeding the $8^{-}$isomeric state in ${ }^{180} \mathrm{Hf}\left(t_{1 / 2}=5.5 \mathrm{hr}\right)$, which then decays to ${ }^{180} \mathrm{Ta}^{\mathrm{m}}$ (Beer \& Ward 1981).
}

cross sections for the $\mathrm{W}$ isotopes are taken from the KADoNiS Database (Dillmann et al. 2006), and correspond to the values published by Macklin et al. (1983) for ${ }^{182} \mathrm{~W}$ and ${ }^{183} \mathrm{~W}$, Marganiec et al. (2009) for ${ }^{184} \mathrm{~W}$ and ${ }^{186} \mathrm{~W}$, and Mohr et al. (2004) for ${ }^{185} \mathrm{~W}$. The models presented here have been selected for $\mathrm{C} / \mathrm{O}>1$ in the envelope so that the condition for the formation of $\mathrm{SiC}$ is satisfied.

In spite of all the differences between the two evolutionary codes, which employ a variety of input physics also concerning mixing and reaction rates (see Cristallo et al. 2009, 2011; Karakas 2010; Karakas et al. 2010 for details), the isotopic compositions predicted by the two sets of models are in fair agreement with one another. Both sets of models present a good match with the $\mathrm{SiC}$ data for the ${ }^{182} \mathrm{~W} /{ }^{184} \mathrm{~W},{ }^{183} \mathrm{~W} /{ }^{184} \mathrm{~W}$, and ${ }^{179} \mathrm{Hf} /{ }^{180} \mathrm{Hf}$ ratios (Figures 6(a), (c) and 7(a), (b), (d), (e)). As already demonstrated by Lugaro et al. (2003) in relation to $\mathrm{Sr}, \mathrm{Zr}$, and $\mathrm{Ba}$ isotopic ratios, the marginal activation of the ${ }^{22} \mathrm{Ne}$ neutron source in the thermal pulses is necessary to best reproduce some of the single $\mathrm{SiC}$ data. In the case of ${ }^{182} \mathrm{~W} /{ }^{184} \mathrm{~W}$ and ${ }^{183} \mathrm{~W} /{ }^{184} \mathrm{~W}$ ratios, the ${ }^{22} \mathrm{Ne}$ neutron source allows the activation of the ${ }^{182} \mathrm{Ta}$ and ${ }^{183} \mathrm{Ta}$ branching points, lowering the ${ }^{182} \mathrm{~W} /{ }^{184} \mathrm{~W}$ and ${ }^{183} \mathrm{~W} /{ }^{184} \mathrm{~W}$ ratios. This is clearly illustrated by comparison in Figures 7 (d) and 7(e) of the $1.8 M_{\odot}$ model, where the ${ }^{22} \mathrm{Ne}$ neutron source is not activated, as the temperature in the thermal pulses is always below $2.66 \times 10^{8} \mathrm{~K}$, with the higher mass models. The $\mathrm{SiC}$ grains that are isotopically solar within errors could be explained either as contamination with solar material, or as originating in a very low mass star (the $1.25 M_{\odot}$ model) where TDU episodes can only slightly modify the envelope's pristine composition. The KJB average measurement can be explained as the average of different stellar masses. Also, the ${ }^{183} \mathrm{~W} /{ }^{184} \mathrm{~W}$ ratio observed in the most extreme grain, LU-41, is slightly lower than the lowest predicted value (but note that the error bars are $1 \sigma$ ).

On the other hand, most models predict lower ${ }^{186} \mathrm{~W} /{ }^{184} \mathrm{~W}$ ratios than those measured in the grains (Figures $6(\mathrm{~b}),(\mathrm{d})$ and 7(c), (f)). The model predictions move toward higher ${ }^{186} \mathrm{~W} /{ }^{184} \mathrm{~W}$ ratios with increasing number of thermal pulses, particularly for models of higher masses and lower metallicities, as the ${ }^{22} \mathrm{Ne}$ neutron source and thus the ${ }^{185} \mathrm{~W}$ branching point are more activated in these models. However, they fail to reach the observed values. While some ad hoc solutions may be found for the single grain data (e.g., the $4 M_{\odot}$ model reaches the ${ }^{186} \mathrm{~W} /{ }^{184} \mathrm{~W}$ ratio measured in grain LU-41 and almost also the value observed in grain LU-36, when considering that the error bars are $1 \sigma$ ), the KJB ratio is particularly puzzling; it agrees with the solar value within a relatively small error while it should represent the average of different stellar sources with different masses and metallicities, or, in case of contamination, should sit on the mixing line connecting the models and the solar composition point.

A question that needs to be addressed is whether or not a revision of the neutron-capture cross sections would improve the match between model predictions and the SiC data. For ${ }^{182} \mathrm{~W}$ and ${ }^{183} \mathrm{~W}$, the uncertainties in the recommended neutron-capture cross sections are very small $(\sim 3 \%)$, but the measurements are quite old (Macklin et al. 1983) and no revision has been obtained since. The possibility that the ${ }^{182} \mathrm{~W}$ neutron-capture cross section had been overestimated by $20 \%-30 \%$ in the past was recently suggested by Wisshak et al. (2006) and Vockenhuber et al. (2007) based on the observation that the ${ }^{182} \mathrm{~W} r$-residual shows a significant positive deviation from the otherwise very smooth $r$-process solar abundance pattern (see 

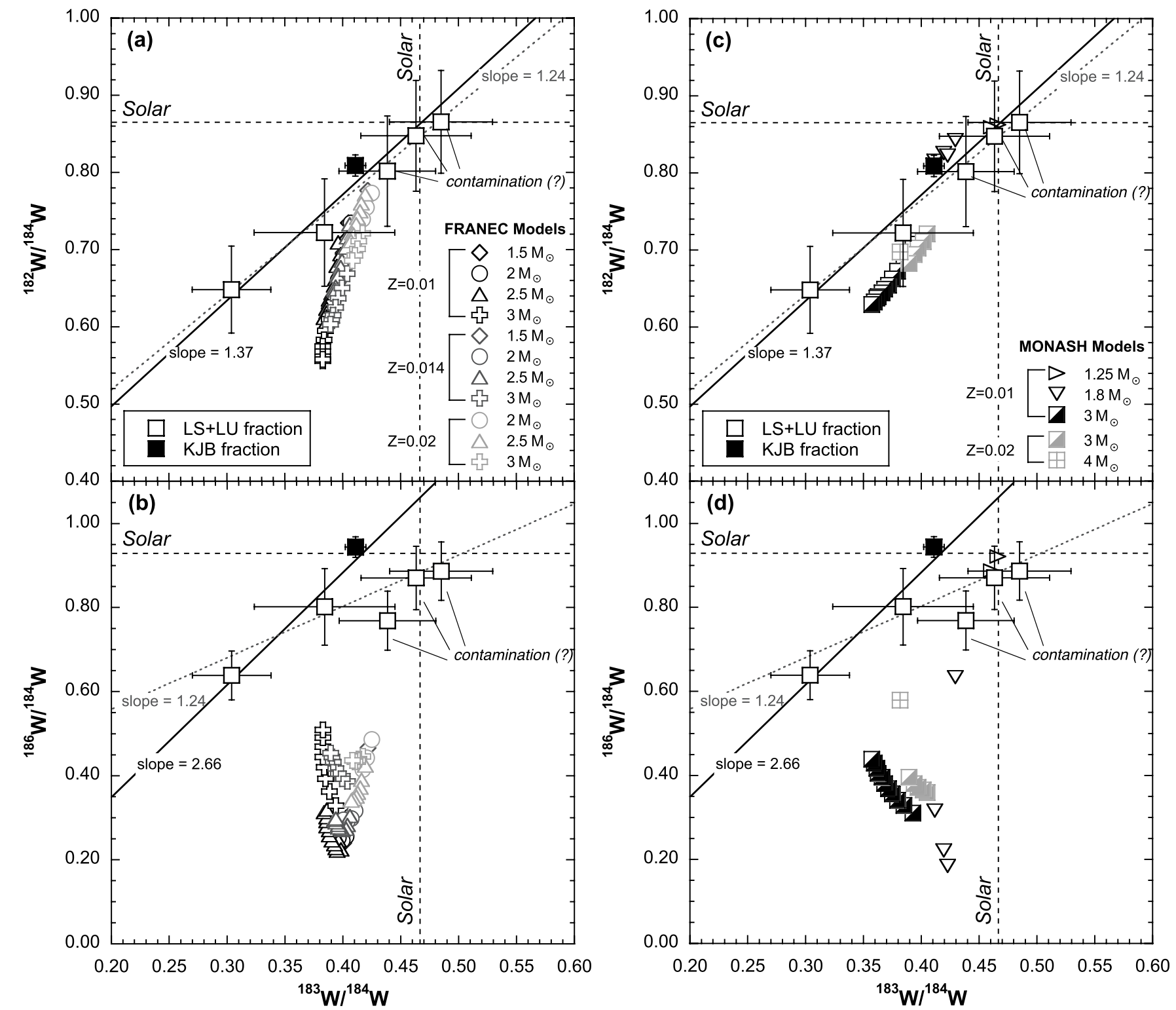

Figure 6. (a) and (c) ${ }^{182} \mathrm{~W} /{ }^{184} \mathrm{~W}$ plotted against ${ }^{183} \mathrm{~W} /{ }^{184} \mathrm{~W}$ and (b) and (d) ${ }^{186} \mathrm{~W} /{ }^{184} \mathrm{~W}$ plotted against ${ }^{183} \mathrm{~W} /{ }^{184} \mathrm{~W}$ for the SiC-enriched sample (KJB fraction) and single $\mathrm{SiC}$ grains (LS+LU fraction). Error bars are $1 \sigma$. The gray dashed lines in each plot give the best linear fit through all single $\mathrm{SiC}$ grains and the SiC-enriched sample. The black solid lines shown in each plot give the best linear fit through grains LU-36 and LU-41, and the SiC-enriched sample; grains LU-34, LU-32, and LU-20 were not included in the fit because their W isotopic signature may be affected by contamination with solar material. Black dashed lines indicate the solar ratios (as inferred from terrestrial composition; Jacobsen 2005). SiC data are compared with $s$-process model predictions (FRANEC and MONASH models) for the envelope compositions of low-mass AGB stars of different masses and metallicities (see the text for details). Predictions are only plotted when the C/O in the stellar envelope reaches values higher than 1.

Figure 12 of Wisshak et al. 2006; or Figure 6 of Vockenhuber et al. 2007). Also, a smaller ${ }^{182}$ Ta neutron-capture cross section, which is based on theoretical calculations with a relatively large uncertainty $(\sim 16 \%)$, could lead to a lower ${ }^{182} \mathrm{Ta}$ branching factor, resulting in a higher $s$-process contribution to ${ }^{182} \mathrm{~W}$ and a lower ${ }^{182} \mathrm{~W} r$-residual (see Table 13 of Vockenhuber et al. 2007). However, any major changes resulting in a higher ${ }^{182} \mathrm{~W}$ $s$-process production would lead to higher ${ }^{182} \mathrm{~W} /{ }^{184} \mathrm{~W}$ ratios and a mismatch with the $\mathrm{SiC}$ data reported here. For example, a $30 \%$ reduction of the ${ }^{182} \mathrm{~W}$ cross section in the MONASH $3 M_{\odot}$ and $Z=0.01$, and $3 M_{\odot}$ and $Z=0.02$ models results in a $\sim 35 \%$ increase in the final ${ }^{182} \mathrm{~W} /{ }^{184} \mathrm{~W}$ ratio in the stellar envelope, which is well above the values observed in the $\mathrm{SiC}$ grains. On the other hand, our data suggest that a small adjustment (toward lower values) in the $s$-process production of ${ }^{183} \mathrm{~W}$ is needed in order to obtain a better agreement between our $\mathrm{SiC}$ data and the $s$-process model predictions. This may be achieved by adopting a higher $(\sim 30 \%){ }^{183} \mathrm{~W}$ neutron-capture cross section.

As for the unstable ${ }^{185} \mathrm{~W}$, its neutron-capture cross section has been recently derived from the inverse ${ }^{186} \mathrm{~W}(\gamma, n)^{185} \mathrm{~W}$ photodisintegration reaction (Sonnabend et al. 2003; Mohr et al. 2004) with an uncertainty between $10 \%$ and $15 \%$ and a difference between the two measurements of $24 \%$ at $30 \mathrm{keV}$. These values lead to an overproduction of the solar $s$-only ${ }^{186} \mathrm{Os}$ of at least 20\% (Sonnabend et al. 2003). Several hypotheses have been raised to resolve this puzzle. Sonnabend et al. (2003) suggested that the stellar model apparently overestimates the $\beta$-decay part and/or underestimates the neutron-capture part of the ${ }^{185} \mathrm{~W}$ branching point. Meyer \& Wang (2007), on the other hand, proposed that this problem could be resolved either by 

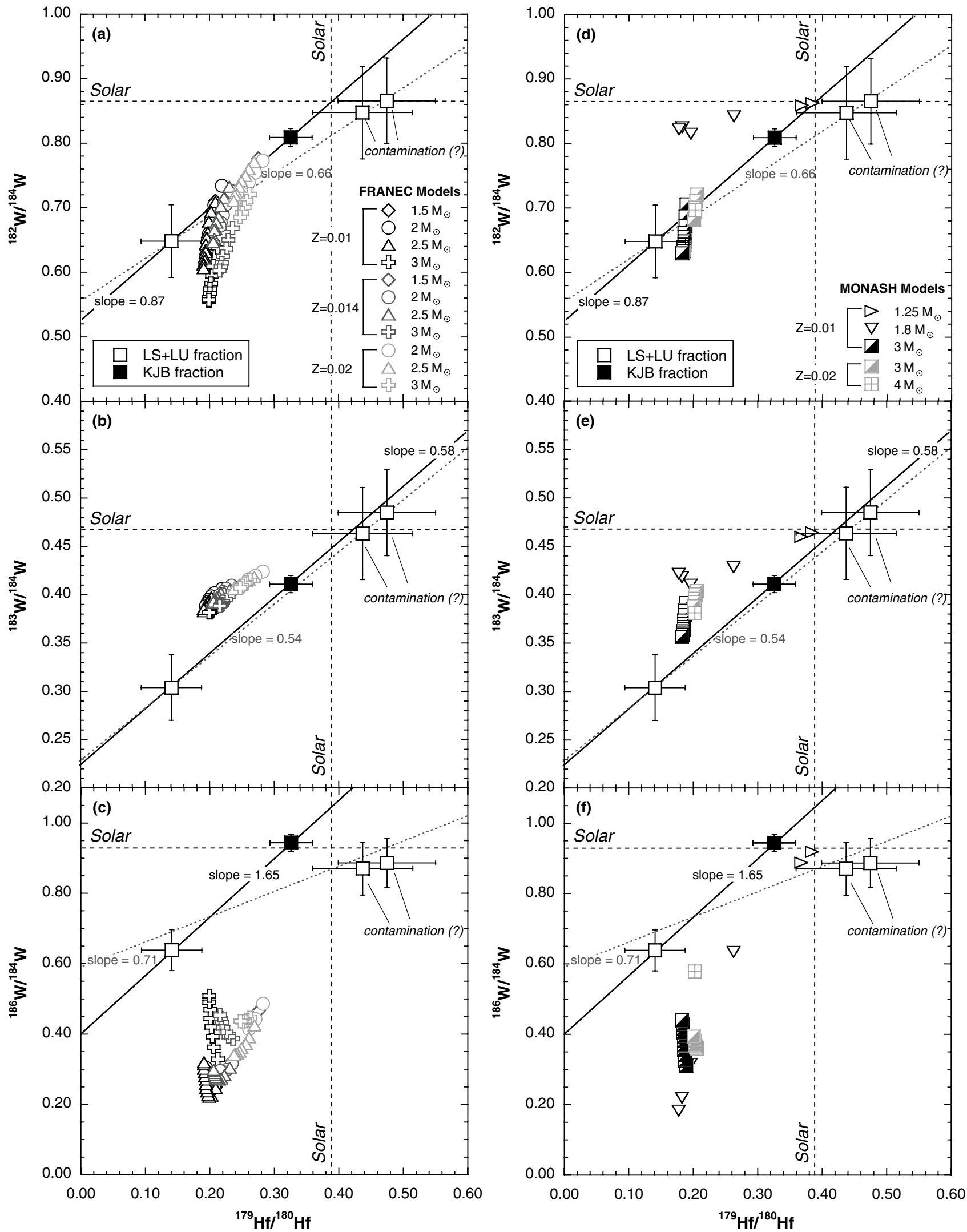

Figure 7. (a) and (d) ${ }^{182} \mathrm{~W} /{ }^{184} \mathrm{~W}$ vs. ${ }^{179} \mathrm{Hf} /{ }^{180} \mathrm{Hf}$, and (b) and (e) ${ }^{183} \mathrm{~W} /{ }^{184} \mathrm{~W}$ vs. ${ }^{179} \mathrm{Hf} /{ }^{180} \mathrm{Hf}$, and (c) and (f) ${ }^{186} \mathrm{~W} /{ }^{184} \mathrm{~W}$ vs. ${ }^{179} \mathrm{Hf} /{ }^{180} \mathrm{Hf}$ for the SiC-enriched sample (KJB fraction) and single SiC grains (LS+LU fraction). Error bars are $1 \sigma$. The gray dashed lines in each plot give the best linear fit through all single SiC grains and the SiC-enriched sample. The black solid lines shown in each plot give the best linear fit through grain LU-41 and the SiC-enriched sample; grains LU-34 and LU-32 were not included in the fit because their W isotopic signature may be affected by contamination with solar material. Black dashed lines indicate the solar ratios (as inferred from terrestrial composition; Jacobsen 2005; Lodders 2010). SiC data are compared with s-process model predictions (FRANEC and MONASH models) for the envelope compositions of low-mass AGB stars of different masses and metallicities (see the text for details). Predictions are only shown when the C/O in the stellar envelope reaches values higher than 1. 
increasing the ${ }^{186}$ Os neutron-capture cross section by $\sim 20 \%$ or by increasing the branching factor at ${ }^{186} \mathrm{Re}$. An increase in the ${ }^{186}$ Os neutron-capture cross section would also help in explaining the low ${ }^{186} \mathrm{Os} /{ }^{188} \mathrm{Os} s$-process ratio derived from isotopic anomalies observed in primitive meteorites (Brandon et al. 2005; Yokoyama et al. 2007). However, a recent measurement of the ${ }^{186}$ Os neutron-capture cross section by Mosconi et al. (2010a) excludes this hypothesis. As noted by Reisberg et al. (2009), the mismatch between the inferred ${ }^{186} \mathrm{Os} /{ }^{188} \mathrm{Os} s$-process ratio, derived from isotopic anomalies observed in primitive meteorites (Brandon et al. 2005; Yokoyama et al. 2007), and model predictions could be resolved by using a recent measurement of the ${ }^{188}$ Os neutron-capture cross section (Mosconi et al. 2007), which is $\sim 27 \%$ lower than the recommended value of Bao et al. (2000). Sonnabend et al. (2003) suggested an increase of the ${ }^{185} \mathrm{~W}$ neutron-capture cross section (by about $60 \%$ ), which would lead to a decrease of ${ }^{186}$ Os and an increase of the ${ }^{186} \mathrm{~W}$ abundance. We computed AGB models using the recommended ${ }^{185} \mathrm{~W}$ neutron-capture cross section multiplied by a factor of two to see if this very large change would help in matching the ${ }^{186} \mathrm{~W} /{ }^{184} \mathrm{~W}$ grain data. We obtain an increase of $\sim 20 \%$ in the final ${ }^{186} \mathrm{~W} /{ }^{184} \mathrm{~W}$ ratio in the stellar envelope of the MONASH $3 M_{\odot}$ and $Z=0.01$, and $3 M_{\odot}$ and $Z=0.02$ models, but it is still lower than the observed stardust $\mathrm{SiC}$ data.

We also checked the impact of the uncertainties in the $\beta$-decay rates of the unstable isotopes ${ }^{181} \mathrm{Hf},{ }^{182} \mathrm{Ta}$, and ${ }^{185} \mathrm{~W}$. At a temperature of $3 \times 10^{8} \mathrm{~K}$, Goriely (1999) reported uncertainties of roughly a factor of three for ${ }^{181} \mathrm{Hf}$ and ${ }^{182} \mathrm{Ta}$ and of roughly $40 \%$ for ${ }^{185} \mathrm{~W}$. Variations were found to be small, at most $20 \%$ in the ${ }^{182} \mathrm{~W} /{ }^{184} \mathrm{~W}$ ratios, mostly due to the uncertainties in the decay rate of ${ }^{182} \mathrm{Ta}$. Further, we checked the impact of the uncertainties in the theoretical neutron-capture cross sections of ${ }^{181} \mathrm{Hf}$ and ${ }^{182} \mathrm{Ta}$ by considering the theoretical estimates reported in the KADoNiS Database (Dillmann et al. 2006) on the basis of different codes used to compute the rates. These uncertainties do not lead to significant variations on the model predictions, at most $16 \%$ in the ${ }^{182} \mathrm{~W} /{ }^{184} \mathrm{~W}$ ratios when the neutron-capture cross section of ${ }^{182}$ Ta was varied by a factor of two.

In summary, the $\mathrm{SiC}$ data do not support the problem of the $s$-process underproduction of ${ }^{182} \mathrm{~W}$ that follows from the high solar $r$-process residual. However, it is important to note that the $r$-process residuals are calculated from $s$-process predictions obtained from one stellar model (or at most from the average of two different masses at the same metallicity; e.g., Arlandini et al. 1999) or from phenomenological models (e.g., Käppeler et al. 1982). A more realistic approach would be to derive them from models of the chemical evolution of the Galaxy (e.g., Travaglio et al. 1999). This is because the solar system composition is the result of nucleosynthesis from many generations of AGB stars of many different masses and metallicities. This more realistic description of the solar $s$-process abundances and of the $r$-process residuals may help solving the discrepancies found in the $\mathrm{Hf}-\mathrm{Ta}-\mathrm{W}-\mathrm{Re}-\mathrm{Os}$ mass region and needs to be pursued.

The SiC data qualitatively support the solution of the ${ }^{186} \mathrm{Os}$ overproduction problem according to which the ${ }^{185} \mathrm{~W}$ branching point should be activated more strongly. However, while the ${ }^{186} \mathrm{Os}$ problem is solved by increasing the neutron-capture cross section of ${ }^{185} \mathrm{~W}$ by $\sim 60 \%$ (Sonnabend et al. 2003), our models do not match the $\mathrm{SiC}$ data even if this cross section is increased by a factor of two. Another way to increase the ${ }^{186} \mathrm{~W} /{ }^{184} \mathrm{~W}$ ratio would be to decrease the production of ${ }^{184} \mathrm{~W}$. The ${ }^{186} \mathrm{~W}$ nuclide is created during thermal pulses, when the
${ }^{185} \mathrm{~W}$ branching point is open. In contrast, ${ }^{184} \mathrm{~W}$ is created within the ${ }^{13} \mathrm{C}$ pocket during the radiative ${ }^{13} \mathrm{C}$ burning. Thus, the production of ${ }^{184} \mathrm{~W}$ is directly correlated with the ${ }^{13} \mathrm{C}$ (and ${ }^{14} \mathrm{~N}$ ) abundances within the pocket, which in turn depend on the mixing mechanism that allows a few protons to penetrate from the envelope during the TDU into the underlying ${ }^{12} \mathrm{C}$ rich intershell. The current uncertainties affecting this mixing are still large, so that a clear description is still not available and the amount of ${ }^{13} \mathrm{C}$ in the pocket may still be treated as a free parameter. In the FRANEC code, the ${ }^{13} \mathrm{C}$ abundance within the pocket is derived from the application of an exponentially decreasing profile of convective velocities at the inner border of the convective envelope. In the MONASH code, the ${ }^{13} \mathrm{C}$ abundance within the pocket is derived from artificial inclusion of an exponentially decreasing abundance of protons in the $\mathrm{He}$ intershell. The inclusion of physical mechanisms not explicitly treated in our codes, such as rotation, could modify the ${ }^{13} \mathrm{C}$ and ${ }^{14} \mathrm{~N}$ abundances within the pocket before and during the activation of the ${ }^{13} \mathrm{C}(\alpha, n){ }^{16} \mathrm{O}$ reaction, thus leading to different ${ }^{186} \mathrm{~W} /{ }^{184} \mathrm{~W}$ ratios. For example, the calculations of Arlandini et al. (1999) used a very different ${ }^{13} \mathrm{C}$ pocket than ours and produced ${ }^{186} \mathrm{~W} /{ }^{184} \mathrm{~W}=0.66$ (together with ${ }^{183} \mathrm{~W} /{ }^{184} \mathrm{~W}=0.35$ ). However, these models were targeted specifically to match the $s$-only solar distribution and are hence not appropriate to be compared to the composition of stardust $\mathrm{SiC}$ grains. In order to test the effects of a different ${ }^{13} \mathrm{C}$ profile in our calculations, we changed in the MONASH $3 M_{\odot}, Z=0.02$ model the extent in mass of the ${ }^{13} \mathrm{C}$ pocket from $0.002 M_{\odot}$ to $0.0005 M_{\odot}$. We obtained ${ }^{186} \mathrm{~W} /{ }^{184} \mathrm{~W}=0.58$, however the ${ }^{183} \mathrm{~W} /{ }^{184} \mathrm{~W}$ ratio is also closer to the solar value (0.43).

Note that although our $\mathrm{SiC}$ data allowed us to address some discrepancies found in the solar $s$-and $r$-abundance distributions in the $\mathrm{Hf}-\mathrm{Ta}-\mathrm{W}-\mathrm{Re}-\mathrm{Os}$ mass region, the stardust grains provide a more direct constraint on the $s$-process in low-mass AGB stars rather than the solar $s$-process component. Furthermore, the calculation of solar $s$ - and $r$-abundance distributions suffers from significant uncertainties. Goriely (1999) used an extended parametric $s$-process model, the "multi-event $s$-process" model described in Goriely (1997), to analyze the impact of nuclear and observational uncertainties on the solar $r$-process residual distribution. The final result, presented in Figure 8 of Goriely (1999), clearly shows that the smoothness of the $r$-process residual curve in the Hf-Ta-W-Re-Os region is well reproduced within the uncertainties. This would still be true when considering the new measurements of the Hf neutron-capture cross sections of Wisshak et al. (2006), which result in a downward shift of the $r$-residual of ${ }^{180} \mathrm{Hf}$. There are other uncertainty factors that may also affect the solar $s$ - and $r$-abundances predictions, like those arising from galactic and stellar evolution models. The combined effect of all uncertainties precludes any definite conclusion about the solar $s$ - and $r$-abundance distributions to be made to the level needed for a detailed comparison with the stardust data.

\section{CONCLUSIONS}

We report the first $\mathrm{W}$ isotopic anomalies in mainstream stardust SiC grains recovered from the Murchison carbonaceous chondrite. Comparisons between grain data and $s$-process model predictions for the envelope compositions of low-mass AGB stars show that a single choice of stellar mass and metallicity cannot account for the range of isotopic ratios observed in the $\mathrm{SiC}$ grains. There is an overall match between the $\mathrm{SiC}$ data and the theoretical predictions for the ${ }^{182} \mathrm{~W} /{ }^{184} \mathrm{~W}$ and 
${ }^{183} \mathrm{~W} /{ }^{184} \mathrm{~W}$ ratios, particularly if a $30 \%$ higher neutron-capture cross section is employed for ${ }^{183} \mathrm{~W}$. However, the AGB models fail to reproduce the high ${ }^{186} \mathrm{~W} /{ }^{184} \mathrm{~W}$ ratios observed in the grains. We note, however, that many uncertainties affect stellar evolutionary computations of the $s$-process. Among them are the formation of the ${ }^{13} \mathrm{C}$ pocket, the mass-loss law, the TDU, and the efficiency of the ${ }^{22} \mathrm{Ne}$ neutron source. For example, the inclusion of rotation and magnetic fields may affect the operation of the ${ }^{13} \mathrm{C}$ neutron source, and a lower efficiency of this neutron source may decrease the production of ${ }^{184} \mathrm{~W}$, thus increasing the ${ }^{183} \mathrm{~W} /{ }^{184} \mathrm{~W}$ ratio. Also a milder mass-loss history would lead to a larger number of thermal pulses and, therefore, possibly to a larger ${ }^{186} \mathrm{~W} /{ }^{184} \mathrm{~W}$ ratio. Similarly, an increase of the ${ }^{22} \mathrm{Ne}(\alpha, n)$ cross section could lead to a higher neutron density, thus increasing the ${ }^{186} \mathrm{~W}$ production, but perhaps worsening the match with the ${ }^{96} \mathrm{Zr} /{ }^{94} \mathrm{Zr}$ ratio observed in the grains (Lugaro et al. 2003). We intend to explore these hypotheses in the future. Finally, further $\mathrm{W}$ isotopic measurements with better precision on additional grains would be extremely helpful. They could shed light on the possibility of contamination affecting the current $\mathrm{W}$ measurements and confirm the high ${ }^{186} \mathrm{~W} /{ }^{184} \mathrm{~W}$ ratios reported here.

J. N. Ávila was supported by a $\mathrm{PhD}$ scholarship (Grant 200081/2005-5) of the Brazilian National Council for Scientific and Technological Development (CNPq). She thanks the Australian National University for an ANU ViceChancellor's Higher Degree Research Travel Grant. M. Lugaro is an ARC Future Fellow and a Monash Research Fellow. T. R. Ireland acknowledges support by ARC grants DP0342772 and DP0666751. E. Zinner acknowledges support by NASA grant NNX08AG71G. S. Cristallo acknowledges support by the Spanish Grant AYA2008-04211-C02-02 and FPA2008-03908 from the MEC. S. Cristallo thanks Roberto Gallino for fruitful scientific discussions. We are grateful to Roy Lewis for providing the LS+LU grains. We thank an anonymous referee for their comments and Eric Feigelson for handling of this manuscript.

\section{REFERENCES}

Amari, S., Hoppe, P., Zinner, E. K., \& Lewis, R. S. 1995, Meteoritics, 30, 679 Amari, S., Lewis, R. S., \& Anders, E. 1994, Geochim. Cosmochim. Acta, 58, 459

Amari, S., Zinner, E. K., \& Lewis, R. S. 2000, Meteor. Planet. Sci., 35, 997

Anders, E., \& Grevesse, N. 1989, Geochim. Cosmochim. Acta, 53, 197

Arlandini, C., Käppeler, F., Wisshak, K., et al. 1999, ApJ, 525, 886

Ávila, J. N., Ireland, T. R., Gyngard, F., et al. 2011, Geochim. Cosmochim. Acta, submitted

Bao, Z. Y., Beer, H., Käppeler, F., et al. 2000, At. Data Nucl. Data Tables, 76, 70

Beer, H., \& Ward, R. A. 1981, Nature, 291, 308

Brandon, A. D., Humayun, M., Puchtel, I. S., Leya, I., \& Zolensky, M. 2005, Science, 309, 1233

Busso, M., Gallino, R., \& Wasserburg, G. J. 1999, ARA\&A, 37, 239

Clayton, D. D. 1983, Principles of Stellar Evolution and Nucleosynthesis (Chicago, IL: Univ. Chicago Press)

Clayton, D. D., \& Nittler, L. R. 2004, ARA\&A, 42, 39

Cristallo, S., Piersanti, L., Straniero, O., et al. 2011, ApJS, accepted (arXiv:1109.1176)

Cristallo, S., Straniero, O., Gallino, R., et al. 2009, ApJ, 696, 797

Cyburt, R. H., Amthor, A. M., Ferguson, R., et al. 2010, ApJS, 189, 240

Dillmann, I., Heil, M., Käppeler, F., et al. 2006, in Capture Gamma-Ray Spectroscopy and Related Topics, ed. A. Woehr \& A. Aprahamian (Melville, NY: AIP), 819, 123

Dodson, M. H. 1978, J. Phys. E: Sci. Instrum., 11, 296

Fujii, K., Mosconi, M., Mengoni, A., et al. 2010, Phys. Rev. C, 82, 015804

Gallino, R., Arlandini, C., Busso, M., et al. 1998, ApJ, 497, 388

Goriely, S. 1997, A\&A, 327, 845
Goriely, S. 1999, A\&A, 342, 881

Gyngard, F., Amari, S., Zinner, E. K., \& Ott, U. 2009a, PASA, 26, 278

Gyngard, F., Amari, S., Zinner, E. K., \& Ott, U. 2009b, ApJ, 694, 359

Hayakawa, T., Shizuma, T., Kajino, T., et al. 2005, ApJ, 628, 533

Heck, P. R., Gyngard, F., Ott, U., et al. 2009, ApJ, 698, 1155

Herwig, F. 2005, ARA\&A, 43, 435

Hoppe, P., Amari, S., Zinner, E. K., Ireland, T., \& Lewis, R. S. 1994, ApJ, 430, 870

Huss, G. R., Ogliore, R. C., Nagashima, K., Telus, M., \& Jilly, C. E. 2011, in Lunar Planet. Sci., Vol. 42, ed. S. J. Mackwell (League City, TX: LPI), abstract 2608

Hynes, K. M., \& Gyngard, F. 2009, in Lunar Planet. Sci., Vol. 40, ed. S. J. Mackwell (League City, TX: LPI), abstract 1198

Ireland, T. R., Zinner, E. K., \& Amari, S. 1991, ApJ, 376, L53

Jacobsen, S. 2005, Annu. Rev. Earth Planet. Sci., 33, 531

Käppeler, F., Arlandini, C., Heil, M., et al. 2004, Phys. Rev. C, 69, 055802

Käppeler, F., Beer, H., Wisshak, K., et al. 1982, ApJ, 257, 821

Karakas, A. I. 2010, MNRAS, 403, 1413

Karakas, A. I., Campbell, S. W., \& Stancliffe, R. J. 2010, ApJ, 713, 374

Kashiv, Y. 2004, PhD thesis, Univ. Chicago

Kinny, P. D., Compston, W., \& Williams, I. S. 1991, Geochim. Cosmochim. Acta, 55, 849

Lodders, K. 2003, ApJ, 591, 1220

Lodders, K. 2010, in Principles and Perspectives in Cosmochemistry, ed. A. Goswami \& B. E. Reddy (Berlin: Springer), 379

Lodders, K., \& Amari, S. 2005, Chem. Erde, 65, 93

Lodders, K., \& Fegley, B. 1995, Meteoritics, 30, 661

Lugaro, M., Davis, A. M., Gallino, R., et al. 2003, ApJ, 593, 486

Macklin, R., Drake, M., \& Arthur, E. 1983, Nucl. Sci. Eng., 84, 98

Marganiec, J., Dillmann, I., Domingo Pardo, C., \& Käppeler, F. 2009, Phys. Rev. C, 80, 025804

Marhas, K. K., Amari, S., Gyngard, F., Zinner, E. K., \& Gallino, R. 2008, ApJ, 689,622

Meyer, B. S., \& Wang, C. 2007, in Lunar Planet. Sci., Vol. 38, ed. S. J. Mackwell (League City, TX: LPI), abstract 2055

Mohr, P., Shizuma, T., Ueda, H., et al. 2004, Phys. Rev. C, 69, 032801

Mosconi, M., Fujii, K., Mengoni, A., et al. 2010a, Phys. Rev. C, 82, 015802

Mosconi, M., Heil, M., Käppeler, F., Plag, R., \& Mengoni, A. 2010b, Phys. Rev. C, 82, 015803

Mosconi, M., Heil, M., Käppeler, F., et al. 2006, in Proc. Int. Symp. on Nuclear Astrophysics, Nuclei in the Cosmos IX, ed. A. Mengoni et al. (PoS NIC-IX; Geneva: CERN), 55

Mosconi, M., Heil, M., Käppeler, F., et al. 2007, Prog. Part. Nucl. Phys., 59, 165

Nicolussi, G. K., Davis, A. M., Pellin, M. J., et al. 1997, Science, 277, 1281

Nicolussi, G. K., Pellin, M. J., Lewis, R. S., et al. 1998a, Geochim. Cosmochim. Acta, 62, 1093

Nicolussi, G. K., Pellin, M. J., Lewis, R. S., et al. 1998b, Phys. Rev. Lett., 81, 3583

Ogliore, R. C., Huss, G. R., \& Nagashima, K. 2011, Nucl. Instrum. Methods: Phys. Res. B, 269, 1910

Qin, L., Dauphas, N., Wadhwa, M., et al. 2008, ApJ, 674, 1234

Reisberg, L., Dauphas, N., Luguet, A., et al. 2009, Earth Planet. Sci. Lett., 277, 334

Savina, M. R., Davis, A. M., Tripa, C. E., et al. 2003, Geochim. Cosmochim. Acta, 67, 3201

Savina, M. R., Davis, A. M., Tripa, C. E., et al. 2004, Science, 303, 649

Segawa, M., Masaki, T., Nagai, Y., et al. 2007, Phys. Rev. C, 76, 022802

Sonnabend, K., Mohr, P., Vogt, K., et al. 2003, ApJ, 583, 506

Straniero, O., Gallino, R., Busso, M., et al. 1995, ApJ, 440, L85

Takahashi, K., \& Yokoi, K. 1987, At. Data Nucl. Data Tables, 36, 375

Travaglio, C., Galli, D., Gallino, R., et al. 1999, ApJ, 521, 691

Virag, A., Wopenka, B., Amari, S., et al. 1992, Geochim. Cosmochim. Acta, 56,1715

Vockenhuber, C., Dillmann, I., Heil, M., et al. 2007, Phys. Rev. C, 75, 015804

Vockenhuber, C., Oberli, F., Bichler, M., et al. 2004, Phys. Rev. Lett., 93, 172501

Wisshak, K., Voss, F., Käppeler, F., et al. 2006, Phys. Rev. C, 73, 045807

Yokoyama, T., Alexander, C. M. O., \& Walker, R. J. 2011, Earth Planet. Sci. Lett., 305, 115

Yokoyama, T., Rai, V., Alexander, C., et al. 2007, Earth Planet. Sci. Lett., 259, 567

Zinner, E. K. 1998, Annu. Rev. Earth Plant. Sci., 26, 147

Zinner, E. K. 2004, in Treatise on Geochemistry, Vol. 1, Meteorites, Planets, and Comets, ed. A. M. Davis, H. D. Holland, \& K. K. Turekian (Oxford: Pergamon), 17 (online update 2007)

Zinner, E. K., Amari, S., \& Lewis, R. S. 1991, ApJ, 382, L47

Zinner, E. K., Nittler, L. R., Gallino, R., et al. 2006, ApJ, 650, 350 